\title{
Cyclic Nucleotide Control of Microtubule Dynamics for Axon Guidance
}

\author{
Hiroki Akiyama, ${ }^{1,2}$ Tetsuko Fukuda, ${ }^{1}$ Takuro Tojima, ${ }^{1,3}$ Viacheslav 0. Nikolaev, ${ }^{4}$ and Hiroyuki Kamiguchi ${ }^{1}$ \\ ${ }^{1}$ Laboratory for Neuronal Growth Mechanisms, RIKEN Brain Science Institute, Wako, Saitama 351-0198, Japan, ${ }^{2}$ Laboratory for Molecular Neurobiology, \\ Faculty of Human Sciences, Waseda University, Tokorozawa, Saitama 359-1192, Japan, ${ }^{3}$ Japan Science and Technology Agency, Kawaguchi, Saitama 332- \\ 0012, Japan, and ${ }^{4}$ Institute of Experimental Cardiovascular Research, University Medical Center Hamburg-Eppendorf, D-20246 Hamburg, Germany
}

Graded distribution of intracellular second messengers, such as $\mathrm{Ca}^{2+}$ and cyclic nucleotides, mediates directional cell migration, including axon navigational responses to extracellular guidance cues, in the developing nervous system. Elevated concentrations of cAMP or cGMP on one side of the neuronal growth cone induce its attractive or repulsive turning, respectively. Although effector processes downstream of $\mathrm{Ca}^{2+}$ have been extensively studied, very little is known about the mechanisms that enable cyclic nucleotides to steer migrating cells. Here, we show that asymmetric cyclic nucleotide signaling across the growth cone mediates axon guidance via modulating microtubule dynamics and membrane organelle transport. In embryonic chick dorsal root ganglion neurons in culture, contact of an extending microtubule with the growth cone leading edge induces localized membrane protrusion at the site of microtubule contact. Such a contact-induced protrusion requires exocytosis of vesicle-associated membrane protein 7 (VAMP7)-positive vesicles that have been transported centrifugally along the microtubule. We found that the two cyclic nucleotides counteractively regulate the frequency of microtubule contacts and targeted delivery of VAMP7 vesicles: cAMP stimulates and cGMP inhibits these events, thereby steering the growth cone in the opposite directions. By contrast, $\mathrm{Ca}^{2+}$ signals elicit no detectable change in either microtubule contacts or VAMP7 vesicle delivery during $\mathrm{Ca}^{2+}$-induced growth cone turning. Our findings clearly demonstrate growth cone steering machinery downstream of cyclic nucleotide signaling and highlight a crucial role of dynamic microtubules in leading-edge protrusion for cell chemotaxis.

Key words: axon guidance; cyclic nucleotide; growth cone; microtubule; VAMP7

\section{Significance Statement}

Developing neurons can extend long axons toward their postsynaptic targets. The tip of each axon, called the growth cone, recognizes extracellular guidance cues and navigates the axon along the correct path. Here we show that asymmetric cyclic nucleotide signaling across the growth cone mediates axon guidance through localized regulation of microtubule dynamics and resulting recruitment of specific populations of membrane vesicles to the growth cone's leading edge. Remarkably, cAMP stimulates microtubule growth and membrane protrusion, whereas cGMP promotes microtubule retraction and membrane senescence, explaining the opposite directional polarities of growth cone turning induced by these cyclic nucleotides. This study reveals a novel microtubule-based mechanism through which cyclic nucleotides polarize the growth cone steering machinery for bidirectional axon guidance.

\section{Introduction}

During development, growth cones at the tips of elongating axons play crucial roles in establishing intricate neural networks.
Guidance cues in the surrounding environment, which are often presented in a form of gradient, activate second-messenger pathways asymmetrically across the growth cone (Tojima et al., 2011; Akiyama and Kamiguchi, 2015). These intracellular signals are translated into asymmetric protrusive activity, causing attractive

construct, A. Miyawaki for VAMP2 and VAMP7 constructs, and Y. Takahashi for Tol 2 and Tol2 transposase constructs. We also thank the RIKEN Brain Science Institute Research Resources Center for providing experimental instruments. The authors declare no competing financial interests.

Correspondence should be addressed to Hiroyuki Kamiguchi, RIKEN Brain Science Institute, 2-1 Hirosawa, Wako, Saitama 351-0198, Japan. E-mail: kamiguchi@brain.riken.jp.

DOI:10.1523/JNEUROSCI.3596-15.2016

Copyright $\odot 2016$ the authors $\quad 0270-6474 / 16 / 365636-14 \$ 15.00 / 0$ 

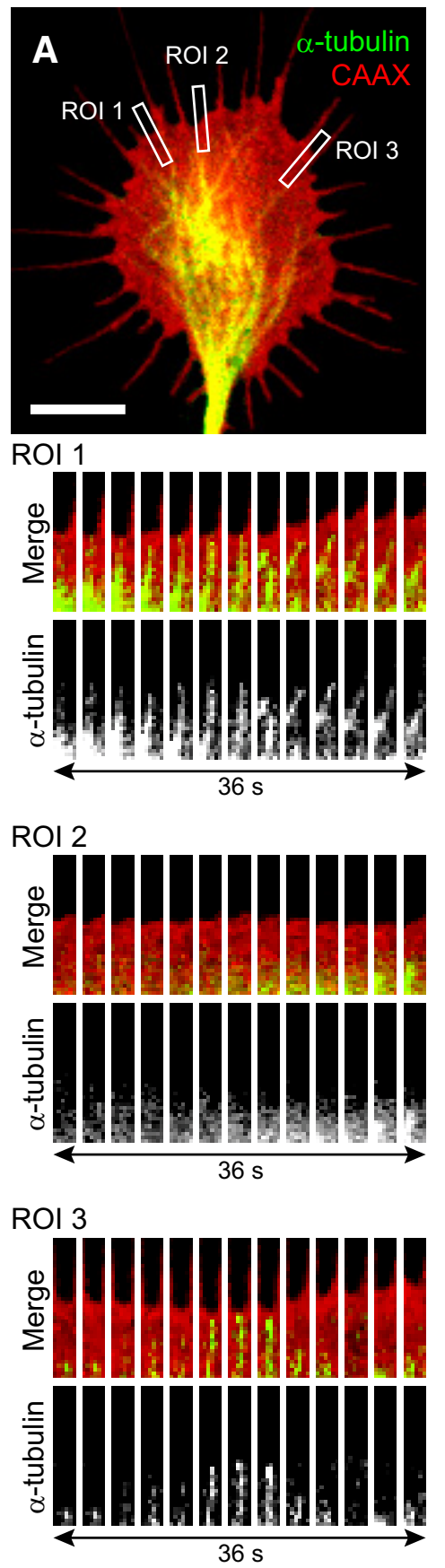

C
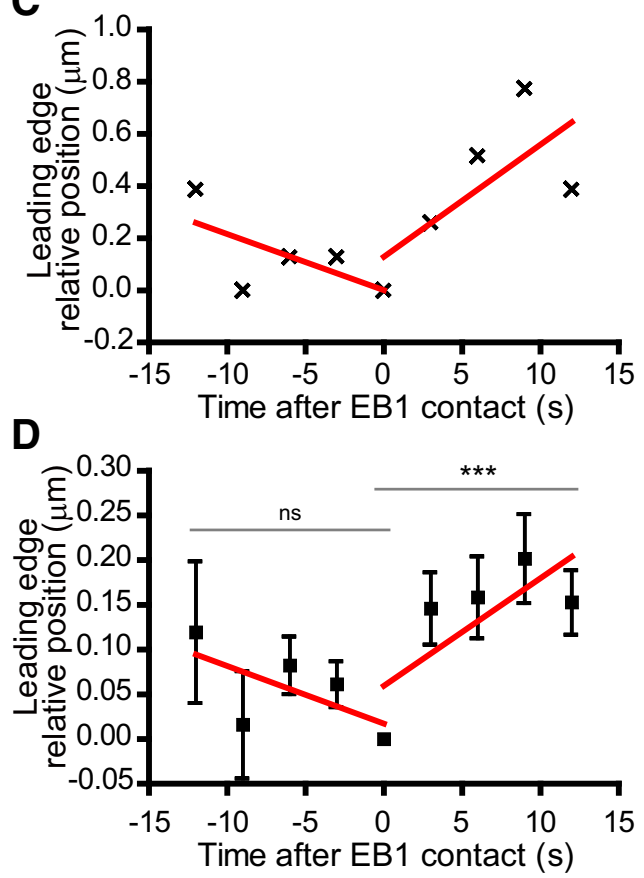

Figure 1. MT contact precedes lamellipodial protrusion. A, A TIRF image of a growth cone expressing EGFP- $\alpha$-tubulin (green) and mCherry-CAAX (red). Lower panels are magnified time-lapse images of ROls 1-3 (white rectangles in the top). The ROIs 1 and 3 represent the leading edge with MT contact, whereas the ROI 2 represents the leading edge without MT contact. B, Fluorescence image of a growth cone expressing EGFP-EB1 (green) and mCherry-CAAX (red). Lower panels are magnified time-lapse views of the white boxed region in the upper panel. Scale bars, $5 \mu \mathrm{m}$. C, The leading-edge position in the white boxed region of the growth cone shown in $\boldsymbol{B}$ was plotted against the time after EB1 contact with the leading edge. The leading-edge position at the time of EB1 contact was set zero, and positive values in the $y$-axis represent edge protrusion. The speed of edge protrusion before and after EB1 contact was estimated by determining the regression lines using five each. $\boldsymbol{D}$, Averaged time course changes of the leading-edge position and the regression lines after EB1 contact in the growth cone shown in $\boldsymbol{B}(n=27$ events). Changes of the leading-edge position were analyzed statistically. ${ }^{* *} p<0.001 ; n s$, not significant, repeated-measures one-way ANOVA.

turning toward or repulsive turning away from the side with guidance signals (Zhou et al., 2002; Myers et al., 2012; San Miguel-Ruiz and Letourneau, 2014). The most-studied second messenger for axon guidance is $\mathrm{Ca}^{2+}$, which mediates both attractive and repulsive turning. Attractive $\mathrm{Ca}^{2+}$ signals induce centrifugal transport and exocytosis of vesicle-associated membrane protein 2 (VAMP2)-positive vesicles to steer growth cones (Tojima et al., 2007; Akiyama and Kamiguchi, 2010). Although microtubules (MTs) are indispensable for this membrane trafficking event, attractive $\mathrm{Ca}^{2+}$ signals do not regulate the dynamics of MTs per se but can increase the frequency of VAMP2 vesicle transport along pre-existing MTs (Tojima et al., 2007). Other second messengers involved in growth cone navigation are the cyclic nucleotides cAMP and cGMP. Many guidance cues elevate intracellular cyclic nucleotide levels in growth cones (Tojima et al., 2011), and asymmetric cyclic nucleotide signaling plays an instructive role in growth cone turning with cAMP inducing attraction and cGMP eliciting repulsion (Lohof et al., 1992; Guirland et al., 2003; Togashi et al., 2008). Because cAMP elevation causes cGMP reduction and vice versa through reciprocal inhibition pathways (Shelly et al., 2010, 2011), it is reasonable to speculate that these messengers counteractively regulate a common effector process for bidirectional axon guidance. However, steering mechanisms downstream of asymmetric cyclic nucleotide signaling remain elusive.

In general, the direction of cell migration depends on the localization of a lamellipodium that defines the cellular front edge (Krause and Gautreau, 2014). Neuronal growth cone turning also relies on a lamellipodium that protrudes and forms an adhesive contact with its new microenvironment forward. Lamellipodial protrusion powered mainly by actin polymerization can expand cytoplasmic space necessary for MT advance that further contributes to protrusion of the edge membrane (Etienne-Manneville, 2013). In addition, MTs may play an instructive role in axon guidance because asymmetric perturbation of MT dynamics in a growth cone is sufficient to initiate turning (Buck and Zheng, 2002). One possible mechanism underlying MT-based lamellipodial protrusion and growth cone turning is targeted delivery of vesicular membranes and associated proteins along the MTs. This notion is consistent with the findings that inhibition of SNARE (soluble $\mathrm{N}$ ethylmaleimide-factor attachment protein receptor) proteins, VAMP2 or VAMP7, causes defects in axon guidance in vitro and in vivo (Tojima et al., 2007; Cotrufo et al., 2011; Zylbersztejn et al., 2012). However, it remains unclear whether and how MTs cause edge membrane protrusion to steer growth cones.

In the present study, we examined the dynamics of MTs and edge membranes simultaneously in growth cones and showed 
that MT contact induces lamellipodial protrusion by supplying vesicular membranes in a VAMP7-dependent manner. We also found differential regulation of these processes by cAMP and cGMP and elucidated a novel mechanism underlying how cyclic nucleotides mediate bidirectional growth cone turning.

\section{Materials and Methods}

Cell culture. Dorsal root ganglion (DRG) neurons from embryonic day 9-10 chicks of either sex were dissociated and cultured on glassbased dishes coated with L1-Fc chimeric proteins that consisted of the whole extracellular domain of L1 and the Fc region of human IgG as described previously (Kamiguchi and Yoshihara, 2001). Cells were maintained in Leibovitz L-15 medium (Invitrogen) supplemented with $\mathrm{N} 2$ (Invitrogen), $750 \mu \mathrm{g} / \mathrm{ml}$ BSA (Invitrogen), and $20 \mathrm{ng} / \mathrm{ml} \mathrm{NGF}$ (Promega) in a humidified atmosphere of $100 \%$ air at $37^{\circ} \mathrm{C}$.

DF-1 cells (ATCC), a cell line derived from embryonic chick fibroblast, were plated on 75 $\mathrm{cm}^{2}$ flasks and cultured in DMEM (Invitrogen) containing $10 \%$ FBS (JRH Biosciences) in a humidified atmosphere of $5 \% \mathrm{CO}_{2} / 95 \%$ air at $37^{\circ} \mathrm{C}$.

Gene transfection. We transfected DRG neurons by electroporation in vitro or in ovo. For in vitro electroporation, we used Nucleofector II (Lonza) or NEPA21 (Nepa Gene) according to the manufacturers' protocols. DRG neurons were transfected with cDNA constructs coding for EGFP (Clontech), TagBFP (Evrogen), mWasabi (Allele Biotechnology), EGFP-end binding protein 1 (EB1; a kind gift from Dr. K. Kaibuchi, Nagoya University, Aichi, Japan), EGFP/mCherry-CAAX (Tojima et al., 2014), mRFP-VAMP2/VAMP7 (a kind gift from Dr. A. Miyawaki, RIKEN Brain Science Institute, Saitama, Japan), pHVenus-VAMP7 (Tojima et al., 2007), TagBFP/mWasabi-VAMP7-longin domain (LD; MartinezArca et al., 2000), Epac2-camps-CAAX (Nikolaev et al., 2004), and cGi500 (Russwurm et al., 2007).

For in ovo electroporation, we used the transposon Tol2 and the Tol2 transposase (a kind gift from Dr. Y. Takahashi, Kyoto University, Kyoto, Japan) to facilitate an integration of the gene of interest into the genome (Sato et al., 2007). The Tol2 vector coding for EGFP- $\alpha$-tubulin (Clontech), mCherry-VAMP7 (a kind gift from Dr. A. Miyawaki), EGFP-EB1, or mCherry-CAAX was injected into the neural tube central canal of a chick embryo at embryonic day 2 (Hamburger-Hamilton stage 10-13). Then, the embryo was electroporated using NEPA2 1 with two electrodes placed parallel to the longitudinal axis of the embryo. The electroporated embryo was incubated for $7 \mathrm{~d}$ before dissecting DRGs.

RNA interference. shRNA against chicken VAMP7 and its control shRNA were provided by OriGene. The shRNA cassette consisted of a 29-mer target gene-specific sequence, a 7-mer loop, and another 29-mer reverse complementary sequence, all under the control of human U6 promoter. The VAMP7-targeting sequence was 5 '-GAGCCATGTGTATGAAGAACCTCAAGCTT- $3^{\prime}$ and the control sequence $5^{\prime}$-GCACTACCAGAGCTAACTCAGATAGTACT- $3^{\prime}$. These shRNA cassettes were subcloned into the Tol2 vector containing an EGFP or TagBFP sequence for identification of shRNA expression. We assessed the efficacy of RNA interference by immunoblotting endogenous VAMP7 expressed in DF-1 cells. DF-1 cells transfected with VAMP7-targeting or control shRNA using NEPA2 1 were incubated for $6 \mathrm{~d}$ and processed for VAMP7 immunoblotting. We also assessed the efficacy of RNA interference in VAMP7-transfected DRG neurons in vitro. Using

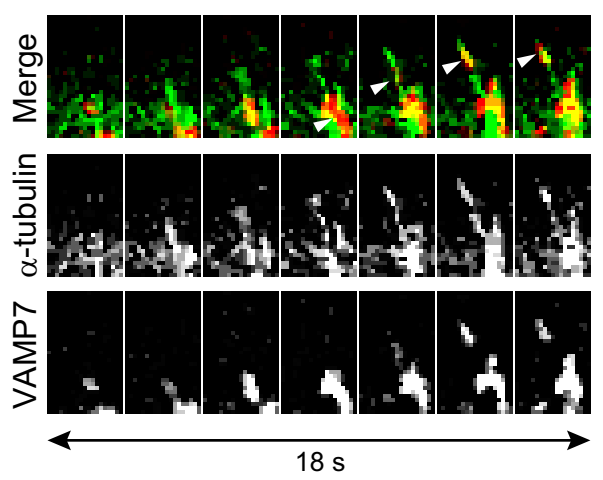

C

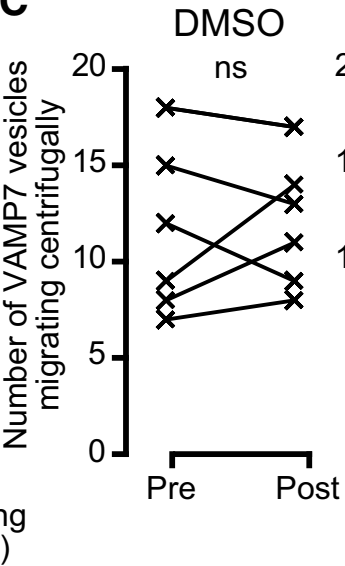

Nocodazole
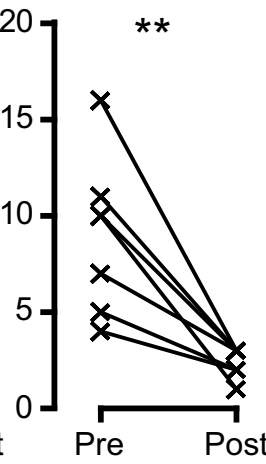

Figure 2. Centrifugal transport of VAMP7 vesicles correlates with MT extension. $A, A$ TIRF image of a growth cone expressing EGP- $\alpha$-tubulin (green) and mCherry-VAMP7 (red). The blue line depicts the growth cone outline. Right panels are magnified the potential effect of different growth cone size, the number of vesicles and comets on this graph had been vesicles migrating centrifugally was counted before (pre) and $10 \mathrm{~min}$ after (post) treatment with DMSO or nocodazole. Each line represent a drug-induced change in a single growth cone. ${ }^{* *} p<0.01$; ns, not significant, paired $t$ test.

NEPA21, DRG neurons were transfected with VAMP7-targeting or control shRNA together with mCherry-tagged chicken VAMP7 of either wild-type or shRNA-resistant sequence. The resistant sequence corresponding to the shRNA targeting site was 5'-GGGCTATGTGCATGAAAAATCTGAAACTG-3', which retained the wild-type amino acid sequence. The cells were then cultured for $2 \mathrm{~d}$ and processed for mCherry immunoblotting.

Immunoblotting. Cultured DF-1 cells were scraped and homogenized, followed by centrifugation at $600 \times g$ for $3 \mathrm{~min}$. The supernatant was centrifuged at $20,400 \times g$ for $60 \mathrm{~min}$, and the resultant pellets were lysed for $60 \mathrm{~min}$ with buffer containing the following: $20 \mathrm{~mm}$ Tris- $\mathrm{HCl}, \mathrm{pH} 7.4$, $300 \mathrm{~mm} \mathrm{NaCl}, 1 \mathrm{~mm}$ dichloro-diphenyl-trichloroethane, 2 mM EGTA, 2 $\mathrm{mm} \mathrm{MgCl}, 2,1 \%$ Triton X-100, 10\% glycerol, protease inhibitor mixture (Complete Mini, Roche). Then, the supernatant obtained after centrifugation at 20,400 $\times g$ for $60 \mathrm{~min}$ was boiled for $5 \mathrm{~min}$ in Laemmli Sample Buffer (Bio-Rad) and processed for SDS-PAGE.

DRG neurons were lysed with N-PER Neuronal Protein Extraction Reagent (Thermo Fisher Scientific) for $30 \mathrm{~min}$ on ice followed by sonication. The supernatant obtained after centrifugation at $20,400 \times g$ for 60 min was boiled for $5 \mathrm{~min}$ in Laemmli Sample Buffer and processed for SDS-PAGE.

We performed SDS-PAGE, and the samples were transferred to a polyvinylidene difluoride membrane (Bio-Rad). The membrane was treated with a blocking solution (Blocking One, Nacalai Tesque) for $60 \mathrm{~min}$ at room temperature and then incubated with mouse anti-VAMP7 monoclonal antibody (1:1000; \#232011, Synaptic Systems) overnight at $4^{\circ} \mathrm{C}$ or with rabbit anti-RFP polyclonal antibody (1:4000; \#600-401-379, Rock- 

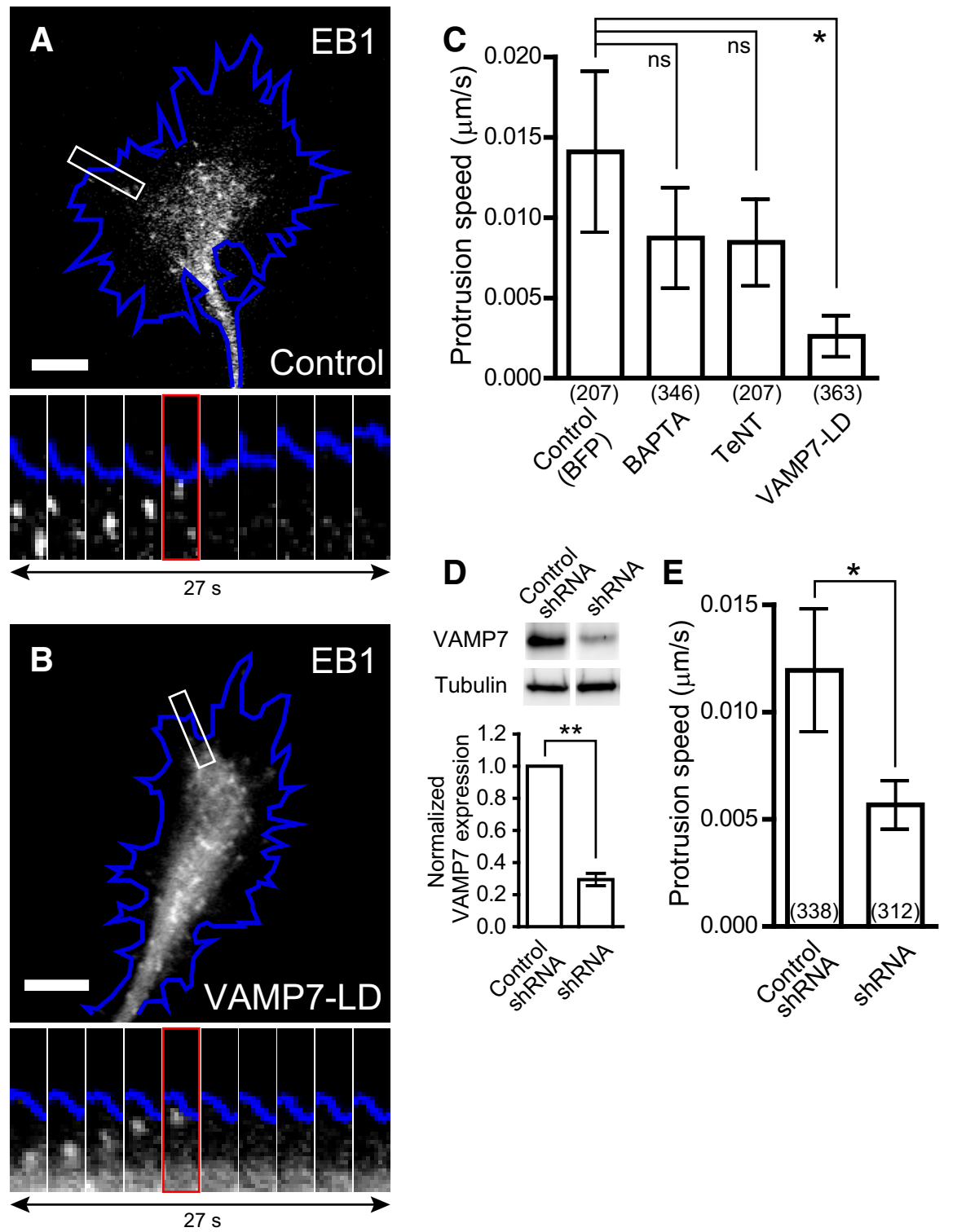

Figure 3. MT-induced lamellipodial protrusion depends on VAMP7. $\boldsymbol{A}, \boldsymbol{B}$, Fluorescence images of EGFP-EB1 in growth cones that coexpress either BFP $(\boldsymbol{A})$ or BFP-tagged VAMP7-LD $(\boldsymbol{B})$. The blue lines depict the growth cone outline. Lower panels are the magnified time-lapse images of white boxed regions in the corresponding upper panels. The red rectangles highlight time-lapse frames of $\mathrm{EB} 1$ contacts with the leading edge. Scale bars, $5 \mu \mathrm{m}$. C, The mean speed of leading-edge protrusion after EB1 contact in growth cones transfected or treated with the indicated constructs or drugs. Numbers in parentheses indicate the number of EB1 contact events examined. ${ }^{*} p<0.05$; ns, not significant, unpaired or Welch's $t$ test with Holm-Bonferroni correction. $D$, The effect of VAMP7 shRNA on endogenous VAMP7 expression in DF-1 cells. Upper panels show immunoblots of VAMP7 and tubulin as a control. A graph represents VAMP7/tubulin normalized to that in control shRNA ( $n=3$ independent experiments). ${ }^{* *} p<0.01$, paired $t$ test. $\boldsymbol{E}$, The mean speed of leading-edge protrusion after EB1 contact in growth cones treated with control or VAMP7 shRNA. ${ }^{*} p<0.05$, Welch's $t$ test.

land Immunochemicals) or mouse anti- $\beta$-tubulin monoclonal antibody (1:3000; \#MAB3408, Millipore) for $60 \mathrm{~min}$ at room temperature. After washing, the membrane was incubated with HRP-conjugated secondary antibody against mouse IgG (1:20,000; \#18881731, Rockland Immunochemicals) or rabbit IgG (1:10,000; \#NA934VS, GE Healthcare). The blots were then visualized with Luminata Forte Western HRP substrate (Millipore). We used ImageQuant LAS4000 (GE Healthcare Life Sciences) for band detection and ImageQuant TL (GE Healthcare Life Sciences) for band quantification. The level of VAMP7 expression was assessed by normalizing the band density of VAMP7 to that of tubulin.

Pharmacological treatment. Membrane-permeable caged cAMP $(2.5 \mu \mathrm{M}$; Invitrogen) or caged cGMP ( $5 \mu \mathrm{m}$; Merck) was applied to neuronal cultures $\geq 30$ min before photolysis experiments. The acetoxymethyl ester of the caged $\mathrm{Ca}^{2+}$ compound $o$-nitrophenyl-EGTA (2 $\mu \mathrm{M}$; Invitrogen) or of BAPTA (1 $\mu \mathrm{M}$ except for Figs. $3 C, 8 A, B$, in which $5 \mu \mathrm{m}$ BAPTA was used; Invitrogen) was loaded into DRG neurons as described previously (Ooashi et al., 2005). Unless otherwise stated, the following reagents were applied to bath media $\geq 30$ min before microscopy or fixation: nocodazole (10 nM; Merck); tetanus neurotoxin (TeNT; 5 nM; List Biological Labs); $8 \mathrm{Br}$-cGMP (1 or $20 \mu \mathrm{M}$, Merck); Sp-cAMPS (1 or $20 \mu \mathrm{M}$; Merck); NF449 (200 $\mu \mathrm{M}$; Merck); ryanodine (100 $\mu \mathrm{M}$; Alomone Labs).

Immunocytochemistry. DRG neurons were fixed at $37^{\circ} \mathrm{C}$ in a solution containing $80 \mathrm{~mm}$ Na-PIPES, pH 6.9, 1 mм $\mathrm{MgCl}_{2}, 1$ mм EGTA, 1 mм GTP, 3\% sucrose, $0.1 \%$ glutaraldehyde, and $4 \%$ formaldehyde (Tojima et al., 2007). The cells were permeabilized with $0.2 \%$ Triton $\mathrm{X}-100$ at room temperature for $60 \mathrm{~min}$ and blocked with $10 \%$ horse serum at room temperature for $30 \mathrm{~min}$. The cells were incubated with anti- $\beta$-tubulin monoclonal antibody (1: 1000; \#MAB3408, Millipore) overnight at $4^{\circ} \mathrm{C}$. Primary antibody binding was visualized with Alexa Fluor-conjugated secondary antibody (10 $\mu \mathrm{g} / \mathrm{ml}$; Invitrogen). Immunofluorescence images were acquired with a $100 \times$ objective (Plan Fluor; numerical aperture, 1.30; Nikon) and a CCD camera (ORCAII-ER with binning set at $1 \times 1$; Hamamatsu Photonics) on an inverted microscope (Eclipse TE300, Nikon). To assess MT distribution, we determined the total length of MTs by summing the length of all individual MTs in the peripheral (P) domain of a single growth cone.

Axon growth assay. DRG neurons transfected with mCherry or mCherry-tagged VAMP7-LD were incubated for $16 \mathrm{~h}$ and processed for axon length measurements as described previously (Kamiguchi and Yoshihara, 2001).

Growth cone turning assay. As described previously (Ooashi et al., 2005; Akiyama et al., 2009), we quantified growth cone turning induced by focal laser-induced photolysis (FLIP) of intracellularly loaded caged compounds or by extracellular microscopic gradients of pituitary adenylate cyclaseactivating polypeptide (PACAP; $5 \mu \mathrm{M}$ in pipette; Peptide Institute) and $\mathrm{PACAP}_{6-38}(5$ $\mu \mathrm{M}$ in pipette; Peptide Institute). Intervals of repetitive FLIP were $10 \mathrm{~s}$ for caged cyclic nucleotides and $3 \mathrm{~s}$ for caged $\mathrm{Ca}^{2+}$.

In some experiments, DRG neurons were transfected in ovo with VAMP7-targeting or control shRNA together with mCherry-tagged shRNA-resistant VAMP7. The embryos were incubated for $7 \mathrm{~d}$ before DRG dissection and growth cone turning assays.

Imaging of MT and membrane dynamics. For Figures $1 A, 2 A, 4 A$, and $6 A-C$, total internal reflection fluorescence (TIRF) images of EGFP- $\alpha$ tubulin, EGFP-EB1, mCherry-VAMP7, EGFP-CAAX, or mCherryCAAX expressed in growth cones were acquired with a $100 \times$ objective (UApo N; numerical aperture, 1.49; Olympus) on an inverted microscope (IX81, Olympus). Green and red fluorescence were detected simultaneously with two CCD cameras (ORCA-AG with binning set at $2 \times 2$; Hamamatsu Photonics) under the control of MetaMorph (version 7.7, MDS Analytical Technologies). The interval of time-lapse imaging was $500 \mathrm{~ms}$ for mCherry-VAMP7 or $3 \mathrm{~s}$ for EGFP- $\alpha$-tubulin and EGFP-EB1. 
TIRF imaging coupled with UV photolysis was performed on a custom-made microscope composed of an upright UV photolysis unit and an inverted TIRF imaging system (Tojima et al., 2010). UV light (330-385 nm) was focused on a circular area of $\sim 10 \mu \mathrm{m}$ in diameter in growth cones through a $40 \times$ water-dipping objective (LUMPlanFl; numerical aperture, 0.8 ; Olympus). Caged cyclic nucleotides were photolyzed every $9 \mathrm{~s}$ by UV (pulse width, 200 $\mathrm{ms}$ ). Caged $\mathrm{Ca}^{2+}$ were photolyzed every $3 \mathrm{~s}$ by UV (pulse width, $100 \mathrm{~ms}$ ).

For Figures $1 B, 2 B, C, 3 A-C, E, 6 D-G$, and $9 A, B$, epifluorescence images of EGFP-EB1, mRFP-VAMP7, mCherry-VAMP7, mRFPVAMP2, EGFP-CAAX, or mCherry-CAAX expressed in growth cones were acquired with a $100 \times$ objective (UplanSApo; numerical aperture, 1.40; Olympus) and a CCD camera (ORCA-ERG or ORCAII-ER with binning set at $2 \times 2$, Hamamatsu Photonics) on an inverted microscope (IX81). The intervals of time-lapse imaging were $3 \mathrm{~s}$ for EB1 and $2 \mathrm{~s}$ for VAMPs. Multicolor images were acquired sequentially using MetaMorph. Guidance signals were produced by repetitive UV photolysis of caged compounds in growth cones or by a PACAP gradient generated with a glass pipette ( $5 \mu \mathrm{M}$ in pipette; positioned at $50 \mu \mathrm{m}$ from the growth cone with $90^{\circ}$ angle with respect to the original direction of axon elongation).

Dynamics of EB1 comets and VAMPpositive vesicles, termed "puncta" in this section, were analyzed using Matlab (2012a, Math Works). We detected and tracked puncta in a growth cone with the Matlab software "plusTipTracker" provided by Dr. G. Danuser's laboratory (Harvard Medical School). Using a custom-made program written with Matlab, we determined the position of the growth cone leading edge as described previously (Akiyama and Kamiguchi, 2014) and analyzed the punctum trajectory to quantify the number of puncta contacting the leading edge. "Contact" was defined as an approach of a centrifugally migrating punctum $<400 \mathrm{~nm}$ from the leading edge. The position of the leading edge was plotted against time, and the speed of edge protrusion was defined as the slope of a regression line from five plots before $(-12$ to $0 \mathrm{~s})$ or after $(0-12 \mathrm{~s})$ the punctum contact. Puncta were excluded from the analysis if their lifetime was $<3$ frames.

Imaging of exocytosis. pHVenus-VAMP7 and mCherry-CAAX in growth cones were excited with 470/22 and 556/20 nm light, respectively. Fluorescence images were acquired every $1 \mathrm{~min}$ with a $100 \times$ objective (UPlanSApo) and a CCD camera (ImagEM $1 \mathrm{~K}$ with binning set at $2 \times 2$, Hamamatsu Photonics) on an inverted microscope (IX81). Multicolor images were acquired sequentially using MetaMorph. The pHVenus/ mCherry emission ratio $(R)$ was used as a measure of VAMP7 exocytosis. Time course changes in VAMP7 exocytosis were expressed as $\Delta R / R_{0}$, where $R_{0}$ is the mean of five consecutive $R$ values before the bath application of folimycin (final concentration of $100 \mathrm{~nm}$; Wako Pure Chemical Industries) and $\Delta R$ represents $R-R_{0}$. VAMP7 exocytosis was evaluated by comparing $\Delta R / R_{0}$ values at 0 and $21 \mathrm{~min}$ after folimycin application.

Imaging of cyclic nucleotides. Epac2-camps-CAAX or cGi-500 in growth cones was excited with $434 / 17 \mathrm{~nm}$ light, and their emission was acquired every $5 \mathrm{~s}$ under the control of MetaMorph. Enhanced cyan fluorescent protein (ECFP) and enhanced yellow fluorescent protein (EYFP) images were simultaneously acquired with a $100 \times$ objective
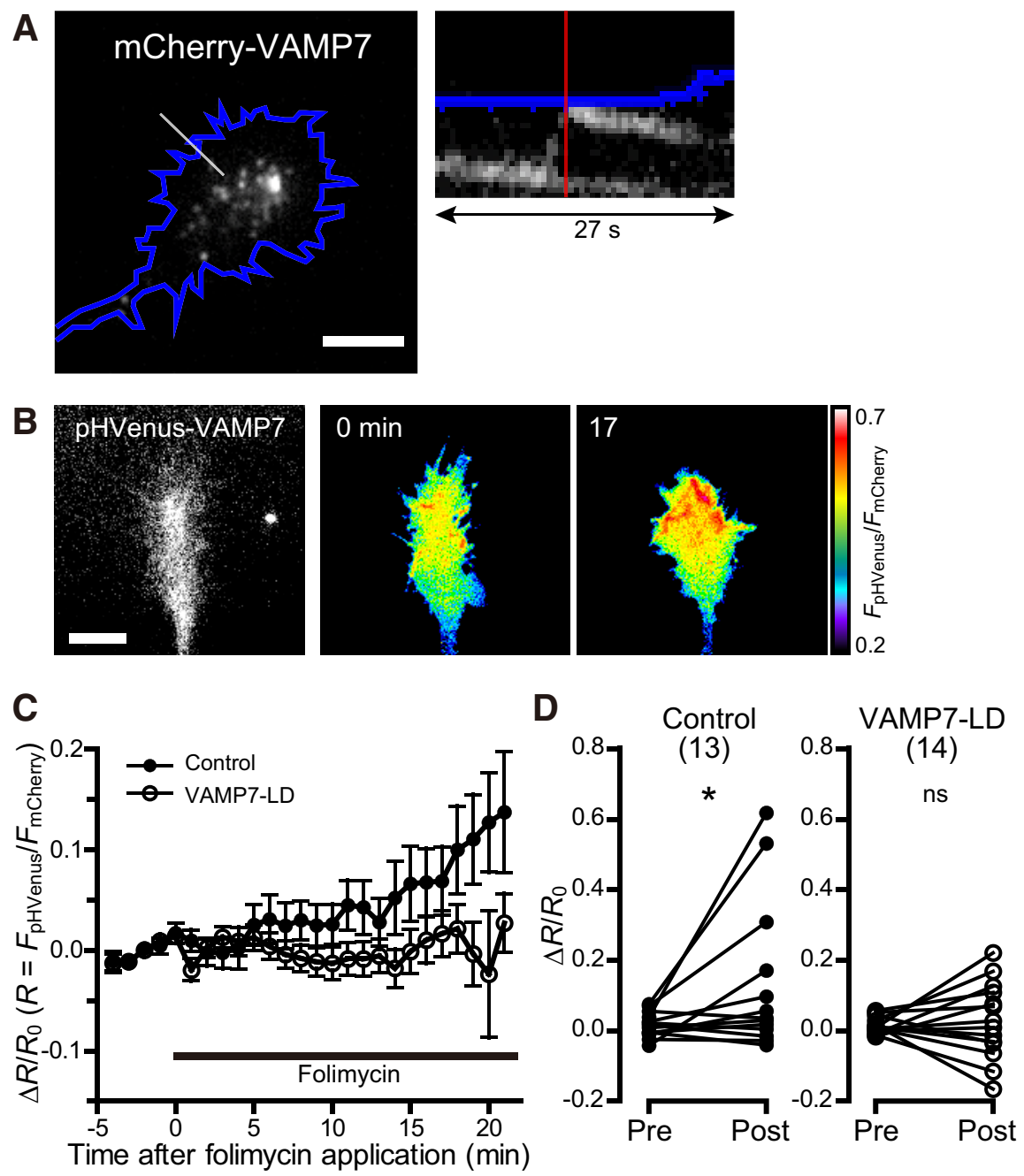

Figure 4. Lamellipodial protrusion upon VAMP7 contact with the leading edge. $\boldsymbol{A}$, A fluorescence image of mCherry-VAMP7 in a growth cone. Right panel is a kymograph along the white line shown in the left panel. The red line in the kymograph corresponds to the timing of VAMP7 contact with the leading edge. Scale bar, $5 \mu \mathrm{m}$. B, A fluorescence image of a growth cone expressing f $R$ values before folimycin application and $\triangle R$ represents $R-R$. and $21 \mathrm{~min}$ (post) after bath application of folimycin. Each line represents folimycin-induced $\Delta R / R_{0}$ changes in a single growth cone. Numbers in parentheses indicate the number of growth cones examined. ${ }^{*} p<0.05$; ns, not significant, paired $t$ test.

(UPlanSApo) and a CCD camera (ORCA-ERG with binning set at $8 \times 8$ or ImagEM $1 \mathrm{~K}$ with binning set at $4 \times 4)$ through an emission splitter (Dual-View, Roper Scientific) equipped with a $505 \mathrm{~nm}$ dichroic mirror, a $475 / 28 \mathrm{~nm}$ filter for ECFP, and a 535/22 filter for EYFP. The ECFP/EYFP emission ratio $(R)$ was used as a measure of cyclic nucleotide concentrations. Time course changes in cyclic nucleotide concentrations were expressed as $\Delta R / R_{0}$, where $R_{0}$ is the mean of 10 consecutive $R$ values before the application of extracellular cues and $\Delta R$ represents $R-R_{0}$. The extracellular cues PACAP and PACAP ${ }_{6-38}$ were applied either to bath media (final concentration of $100 \mathrm{nM}$ ) or as a gradient ( $5 \mu \mathrm{M}$ in pipette; positioned at $50 \mu \mathrm{m}$ from the growth cone with $90^{\circ}$ angle with respect to the original direction of axon elongation). As a measure of the amplitude of cyclic nucleotide elevations, we calculated the mean of three consecutive $\Delta R / R_{0}$ values that included, in the middle, the maximum $\Delta R / R_{0}$ after bath application of PACAP/PACAP ${ }_{6-38}$, or the mean of $\Delta R / R_{0}$ during the period from 1 to $7.5 \mathrm{~min}$ after the onset of a PACAP/PACAP ${ }_{6-38}$ gradient. To assess an asymmetry in cyclic nucleotide elevations across the growth cone, we positioned ROIs on both sides of the growth cone as described previously (Akiyama and Kamiguchi, 2014): "near ROI" on the side fac- 

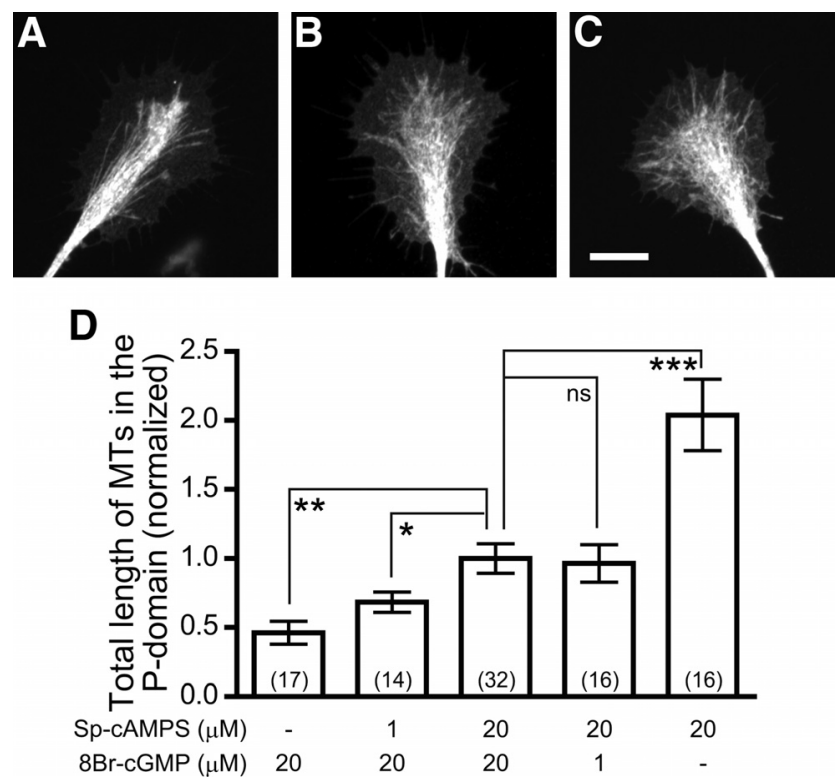

Figure 5. CAMP and CGMP counteractively regulate MT distribution into the P domain. $\boldsymbol{A}-\boldsymbol{C}$, Immunofluorescence showing MT distribution in growth cones treated for 30 min with $20 \mu \mathrm{M}$ $8 \mathrm{Br}$-cGMP (A), $20 \mu \mathrm{m} 8 \mathrm{Br}$-cGMP and $20 \mu \mathrm{m}$ Sp-CAMPS (B), or $20 \mu \mathrm{m}$ Sp-CAMPS (C). Scale bar, 10 $\mu \mathrm{m}$. $\boldsymbol{D}$, The total length of MTs in the P domain of growth cones cotreated with the indicated doses of Sp-cAMPS and 8Br-cGMP. Numbers in parentheses indicate the number of growth cones examined. ${ }^{*} p<0.05,{ }^{* *} p<0.01,{ }^{* *} p<0.001$; ns, not significant, unpaired or Welch's $t$ test with Holm-Bonferroni correction.

ing PACAP/PACAP ${ }_{6-38}$ source and "far ROI" on the opposite side. Then, time course changes in $R^{\prime}$ (designated as $\Delta R^{\prime} / R^{\prime}{ }_{0}$ ) were determined, where $R^{\prime}$ is a ratio of $R$ in the near ROI to that in the far ROI. The mean of $\Delta R^{\prime} / R^{\prime}{ }_{0}$ during the period from 1 to $7.5 \mathrm{~min}$ after the onset of a $\mathrm{PACAP} \mathrm{PACAP}_{6-38}$ gradient was calculated to assess cue-induced asymmetry in cyclic nucleotide elevations.

Statistics. Data are expressed as the mean \pm SEM. Statistical analyses were performed using Prism (version 4.01, GraphPad). We performed paired or unpaired $t$ tests to compare the mean difference of two groups. Three or more groups were compared using Holm-Bonferronicorrected $t$ test or Dunnett's multiple-comparison test, except for Figure $1 D$ where we used repeated measures one-way ANOVA. $p<0.05$ was judged statistically significant.

\section{Results}

\section{MT contact precedes lamellipodial protrusion}

We examined the dynamics of MTs and lamellipodial membranes in growth cones expressing EGFP- $\alpha$-tubulin and mCherry-CAAX (Fig. 1A). MTs in the growth cone central (C) domain occasionally extended into the $\mathrm{P}$ domain and reached the leading edge. Following their contacts with the edge, the lamellipodia at the site of MT contacts moved forward. We also visualized MT dynamics using EGFP-EB1, which associates with the actively polymerizing plus-end of MTs (Mimori-Kiyosue et al., 2000; Akhmanova and Steinmetz, 2008), and found that polymerizing MTs reached the edge and then induced localized lamellipodial protrusion (Fig. $1 B-D$ ). We quantified the speed of leading-edge protrusion before and after MT tip contact and found a statistically significant difference $(-0.010 \pm 0.0022$ $\mu \mathrm{m} / \mathrm{s}$ before contact vs $0.014 \pm 0.0050 \mu \mathrm{m} / \mathrm{s}$ after contact, $p<$ 0.0001 , paired $t$ test, $n=207$ events in 10 growth cones).

\section{MT-induced lamellipodial protrusion depends on VAMP7}

We examined the dynamics of intracellular vesicular membranes to test whether the protrusive activity of MTs depends on organ- elle transport along them. Simultaneous imaging of EGFP- $\alpha$ tubulin and mCherry-VAMP7 revealed that VAMP7-positive vesicles migrated along dynamic MTs toward the leading edge (Fig. 2A). The number of VAMP7 vesicles migrating into the $\mathrm{P}$ domain showed a positive correlation with the number of EB1positive MT plus-ends advancing into the $\mathrm{P}$ domain [Fig. 2B; Pearson's correlation coefficient $(r)=0.83 ; p<0.0001]$. After treating growth cones with the MT destabilizer nocodazole, fewer VAMP7 vesicles migrated into the $\mathrm{P}$ domain (Fig. $2 C$ ), which is consistent with the previous finding that MTs serve as a rail for transporting VAMP7 vesicles (Burgo et al., 2012).

Next, to examine the involvement of VAMP7 in MT-induced lamellipodial protrusion, we used the N-terminal autoinhibitory domain of VAMP7 (VAMP7-LD), which inhibits VAMP7mediated exocytosis by blocking VAMP7 interaction with SNAP-25 (Martinez-Arca et al., 2000). Consistent with a previous report (Martinez-Arca et al., 2000), VAMP7-LD inhibited axon growth [100 $\pm 8.5 \%$ (94 control axons) vs $52 \pm 4.7 \%$ (115 VAMP7-LD-expressing axons), $p<0.0001$, unpaired $t$ test]. MT contacts induced lamellipodial protrusion but failed to do so in growth cones expressing VAMP7-LD (Fig. 3A-C). To test for the involvement of VAMP7 more specifically, we designed and synthesized an shRNA that decreased the expression of chicken VAMP7 (Fig. 3D) but not VAMP2 (data not shown). This VAMP7 shRNA inhibited local lamellipodial protrusion after MT contact (Fig. 3E). However, MT-induced lamellipodial protrusion was not affected by TeNT, a toxin that cleaves VAMP2, or by BAPTA-AM, a $\mathrm{Ca}^{2+}$ chelator (Fig. 3C). We further showed that lamellipodial protrusion occurs after the arrival of VAMP7 vesicles (Fig. 4A). There was a significant difference in the mean speed of leading-edge protrusion before and after VAMP7 vesicle contacts $(-0.0069 \pm 0.0033 \mu \mathrm{m} / \mathrm{s}$ for before contact vs $0.015 \pm$ $0.0072 \mu \mathrm{m} / \mathrm{s}$ for after contact, $p<0.01$, paired $t$ test, $n=174$ events in 20 growth cones). Because these data suggested VAMP7-mediated fusion of vesicle membranes with plasmalemma, we monitored VAMP7 exocytosis by measuring fluorescence of pHVenus, a pH-sensitive version of Venus (Tojima et al., 2007), attached to the luminal end of VAMP7. To detect constitutive membrane fusion, the vacuolar ATPase inhibitor folimycin is often used to amplify fluorescence signals because exocytosed vesicles cannot be reacidified in the presence of this drug (Atasoy et al., 2008; Hua et al., 2011; Bal et al., 2013). After the bath application of folimycin, control but not VAMP7-LDexpressing growth cones showed a significant increase in $\mathrm{pHVe}$ nus fluorescence (Fig. 4B-D). Collectively, these results indicate that lamellipodial protrusion depends on MT-guided delivery and exocytosis of VAMP7 vesicles.

\section{cAMP and cGMP counteractively regulate MT-guided transport of VAMP7 vesicles}

To test whether cyclic nucleotides regulate MT dynamics, we first examined MT distribution in the growth cone $\mathrm{P}$ domain after treatment with differential combinations of cAMP and cGMP analogs (Fig. 5). These analogs had counteractive effects on MT distribution: cAMP and cGMP increased and decreased the total length of MTs in the growth cone P domain, respectively (Fig. 5A-D).

We next examined the effect of spatially restricted cyclic nucleotide signals on MT dynamics. We photolyzed caged cAMP or cGMP on one side of growth cones expressing EGFP-EB1 and mCherry-CAAX and quantified the frequency of MT contacts with the leading edge on the photolysis side (near) and the opposite side (far; Fig. 6A). The photolysis-induced generation of 

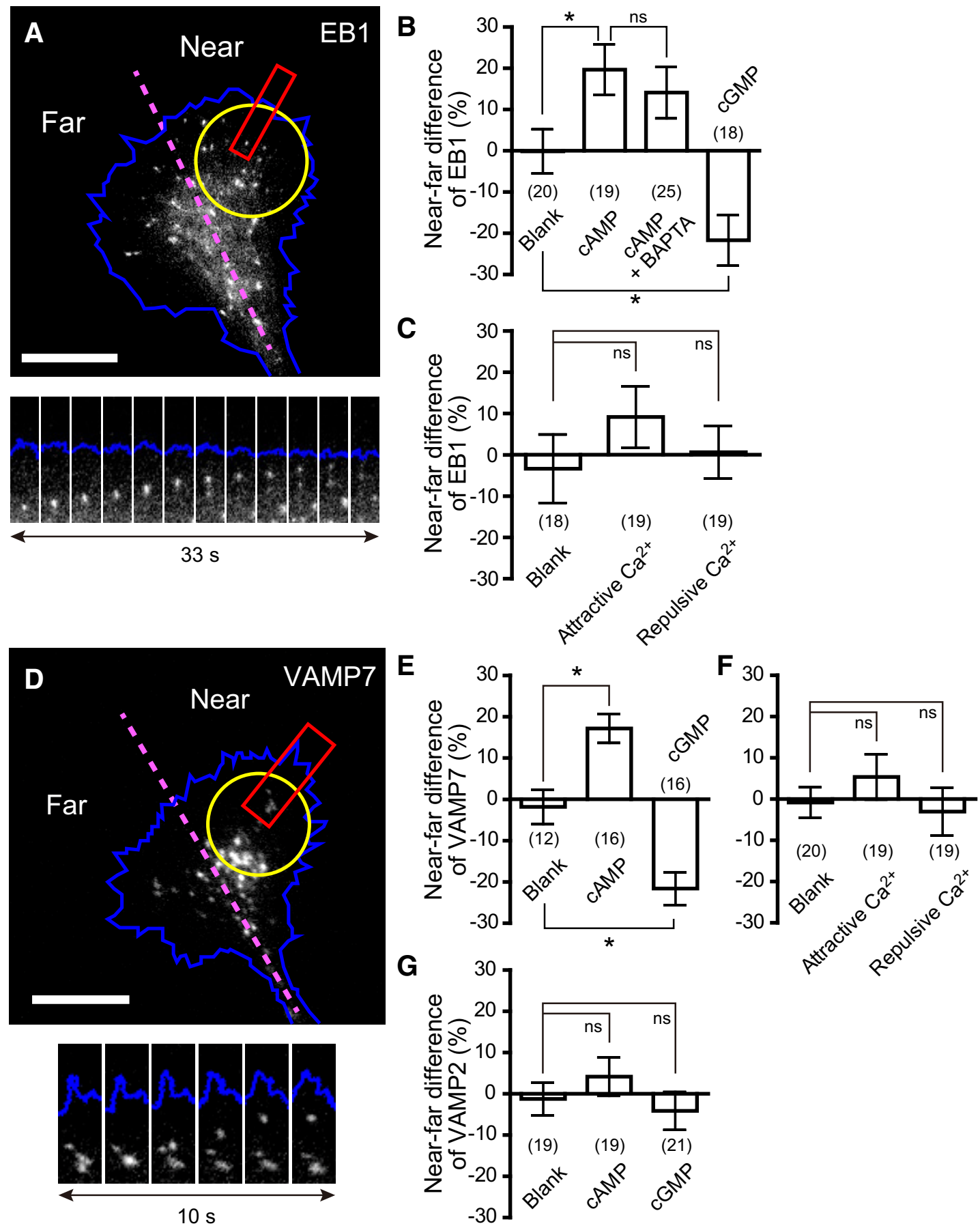

Figure 6. CAMP and CGMP counteractively regulate MT-guided transport of VAMP7 vesicles. A, A TIRF image of EGFP-EB1 in a growth cone outlined in blue. Caged cAMP was photolyzed by UV irradiation (yellow circle), and the behavior of EB1 comets was analyzed on the UV-irradiated side (Near) and the opposite side (Far). The pink broken line indicates the boundary between Near and Far. Lower panels are the magnified time-lapse images of the red boxed region in the upper panel. $\boldsymbol{B}, \boldsymbol{C}$, The effect of photolysis-induced cyclic nucleotide $(\boldsymbol{B})$ and $\mathrm{Ca}^{2+}(\boldsymbol{C})$ signals on $\mathrm{MT}$ extension in growth cones. The number of EB1 comets contacting the leading edge on the near or far side was divided by the length of the C-domain/P-domain boundary on the corresponding side. Shown is the mean of near-far differences of EB1 contact frequency calculated as (near - far)/(near $+\mathrm{far}$ ) $\times 100$, with positive and negative values indicating more frequent EB1 contact on the near and far sides, respectively. Caged compound loading was omitted in "Blank." Numbers in parentheses indicate the number of growth cones examined. ${ }^{*} p<0.05$; ns, not significant; $\boldsymbol{B}$, unpaired $t$ test with Holm-Bonferroni correction; C, Dunnett's multiple-comparison test. $\boldsymbol{D}$, A fluorescence image of mRFP-VAMP7 in a growth cone. The behavior of VAMP7 vesicles was analyzed after photolyzing caged cAMP (yellow circle). The pink broken line indicates the boundary between Near and Far. Lower panels are the magnified time-lapse images of the red boxed region in the upper panel. Scale bars, $10 \mu \mathrm{m}$. The number of VAMP7 $(\boldsymbol{E}, \boldsymbol{F})$ or VAMP2 $(\boldsymbol{G})$ vesicles migrating centrifugally on either side was counted and divided by the length of C-domain/P-domain boundary. ${ }^{*} p<0.05 ; n s$, not significant, Dunnett's multiple-comparison test.

cAMP signals increased the MT contact frequency on the near side even when growth cones had been loaded with BAPTA-AM (Fig. 6B), indicating that cAMP facilitates MT advance independently of $\mathrm{Ca}^{2+}$. Conversely, cGMP decreased the MT contact frequency on the near side (Fig. 6B). We also examined the effects of $\mathrm{Ca}^{2+}$ signals on MT dynamics. As reported previously (Ooashi et al., 2005; Tojima et al., 2009), photolyzing caged $\mathrm{Ca}^{2+}$ on one side of a growth cone induces attraction under the culture con- 

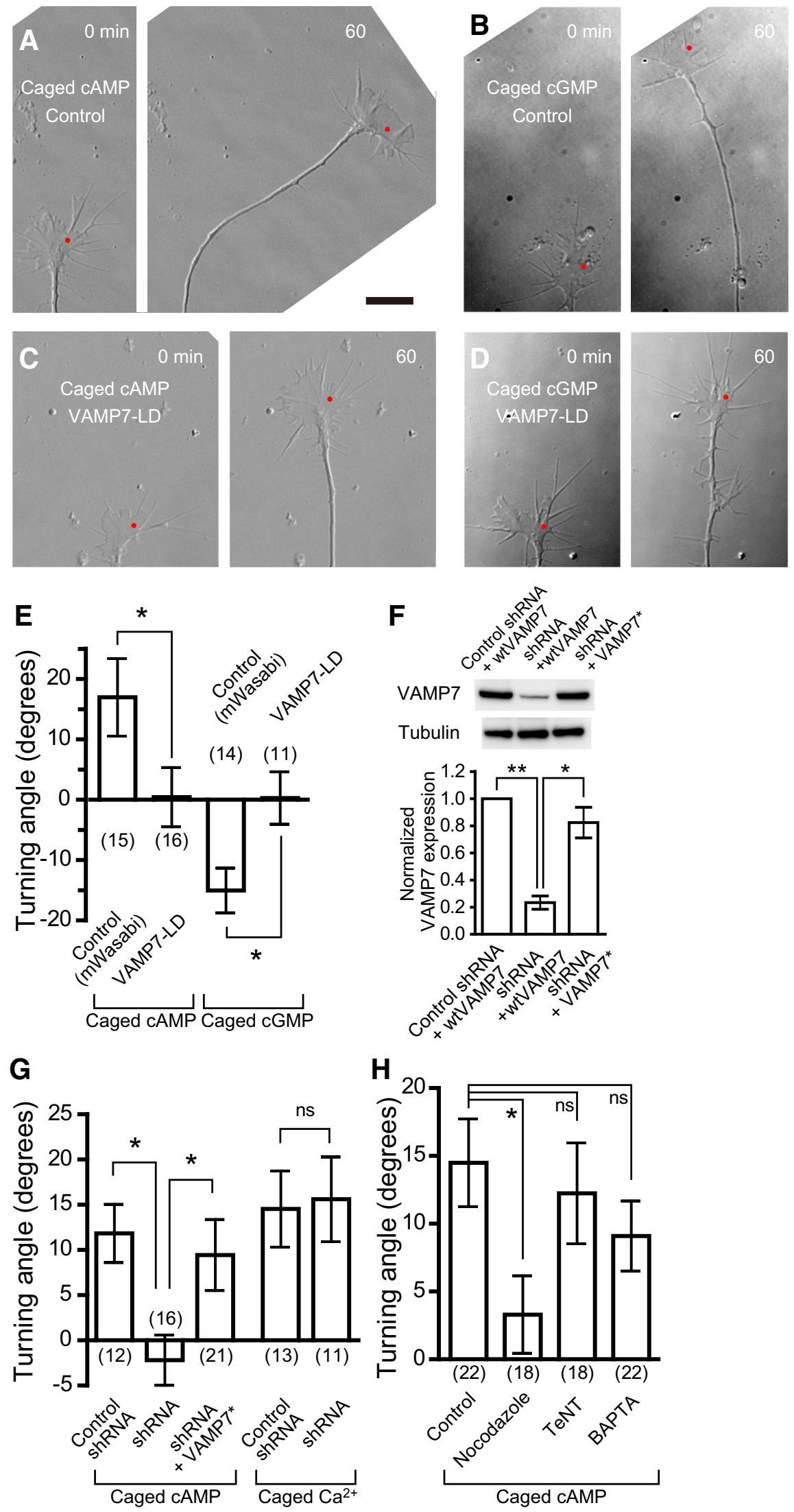

Figure 7. VAMP7 is necessary for cyclic nucleotide-induced growth cone turning. $\boldsymbol{A}-\boldsymbol{D}$, Time-lapse differential interference contrast images of growth cones transfected with mWasabi $(\boldsymbol{A}, \boldsymbol{B})$ or mWasabi-tagged VAMP7-LD $(\boldsymbol{C}, \boldsymbol{D})$ constructs and then loaded with caged CAMP $(\boldsymbol{A}, \boldsymbol{C})$ or caged $\operatorname{cGMP}(\boldsymbol{B}, \boldsymbol{D})$. Caged compounds were photolyzed by repetitive FLIP on one side of the growth cone (red spots). Digits represent minutes after the start of repetitive FLIP. Scale bar, $10 \mu \mathrm{m}$. $\boldsymbol{E}$, The average angle of growth cone turning induced by FLIP of caged CAMP or cGMP. The growth cones express either mWasabi (control) or dition used in this study but causes repulsion if secondary $\mathrm{Ca}^{2+}$ release from the endoplasmic reticulum is blocked with a high dose of ryanodine, an inhibitor of ryanodine receptors. Therefore we photolyzed caged $\mathrm{Ca}^{2+}$ in growth cones pretreated with or without ryanodine to generate repulsive or attractive $\mathrm{Ca}^{2+}$ signals, respectively. Consistent with previous work (Tojima et al., 2007), neither repulsive nor attractive $\mathrm{Ca}^{2+}$ signals caused a significant asymmetry in the frequency of MT contacts (Fig. 6C). These results demonstrate that cyclic nucleotides but not $\mathrm{Ca}^{2+}$ regulate MT dynamics in growth cones.

We then analyzed MT-guided transport of membrane vesicles in growth cones (Fig. $6 D-G$ ). Photolysis-induced cAMP or cGMP signals increased or decreased, respectively, the number of VAMP7 vesicles migrating into the $\mathrm{P}$ domain on the near side (Fig. 6E). By contrast, $\mathrm{Ca}^{2+}$ signals had no detectable effect on the transport of VAMP7 vesicles (Fig. $6 F)$. Because it is known that the centrifugal transport of VAMP2 vesicles drives growth cone attraction in response to $\mathrm{Ca}^{2+}$ signals (Tojima et al., 2007), we tested whether cyclic nucleotides also act on VAMP2 vesicles. Neither cAMP nor cGMP caused an asymmetry in the number of VAMP2 vesicles migrating centrifugally (Fig. $6 G$ ). These results strongly suggest that cyclic nucleotides and $\mathrm{Ca}^{2+}$ use distinct machinery for growth cone turning, with cyclic nucleotides regulating the transport of VAMP7 vesicles and $\mathrm{Ca}^{2+}$ acting on VAMP2 vesicles.

\section{VAMP7 is necessary for cyclic nucleotide-induced growth cone turning}

We tested whether VAMP7 is necessary for growth cone turning induced by cyclic nu-

\section{$\leftarrow$}

mWasabi-tagged VAMP7-LD. Positive and negative values on the $y$-axis indicate attractive and repulsive turning, respectively. Numbers in parentheses indicate the number of growth cones examined. ${ }^{*} p<0.05$, unpaired $t$ test. $\boldsymbol{F}$, The effect of VAMP7 ShRNA on the expression of exogenous VAMP7 of wildtype (wtVAMP7) or shRNA-resistant sequence (VAMP7*) in DRG neurons. Upper panels show immunoblots of VAMP7 and tubulin as a control. A graph represents VAMP7/tubulin normalized to that in control shRNA ( $n=3$ independent experiments). ${ }^{*} p<0.05,{ }^{* *} p<0.01$, paired $t$ test with Holm-Bonferroni correction. $\boldsymbol{G}, \boldsymbol{H}$, The average angle of growth cone turning induced by FLIP of caged CAMP or caged $\mathrm{Ca}^{2+}$. The growth cones were genetically or pharmacologically pretreated as indicated. ${ }^{*} p<0.05$, Dunnett's multiplecomparison test (G, caged (AMP); ns, not significant, unpaired $t$ test $\left(G\right.$, caged $\left.\mathrm{Ca}^{2+}\right),{ }^{*} p<0.05$; ns, not significant, Dunnett's multiple-comparison test $(\boldsymbol{H})$. 

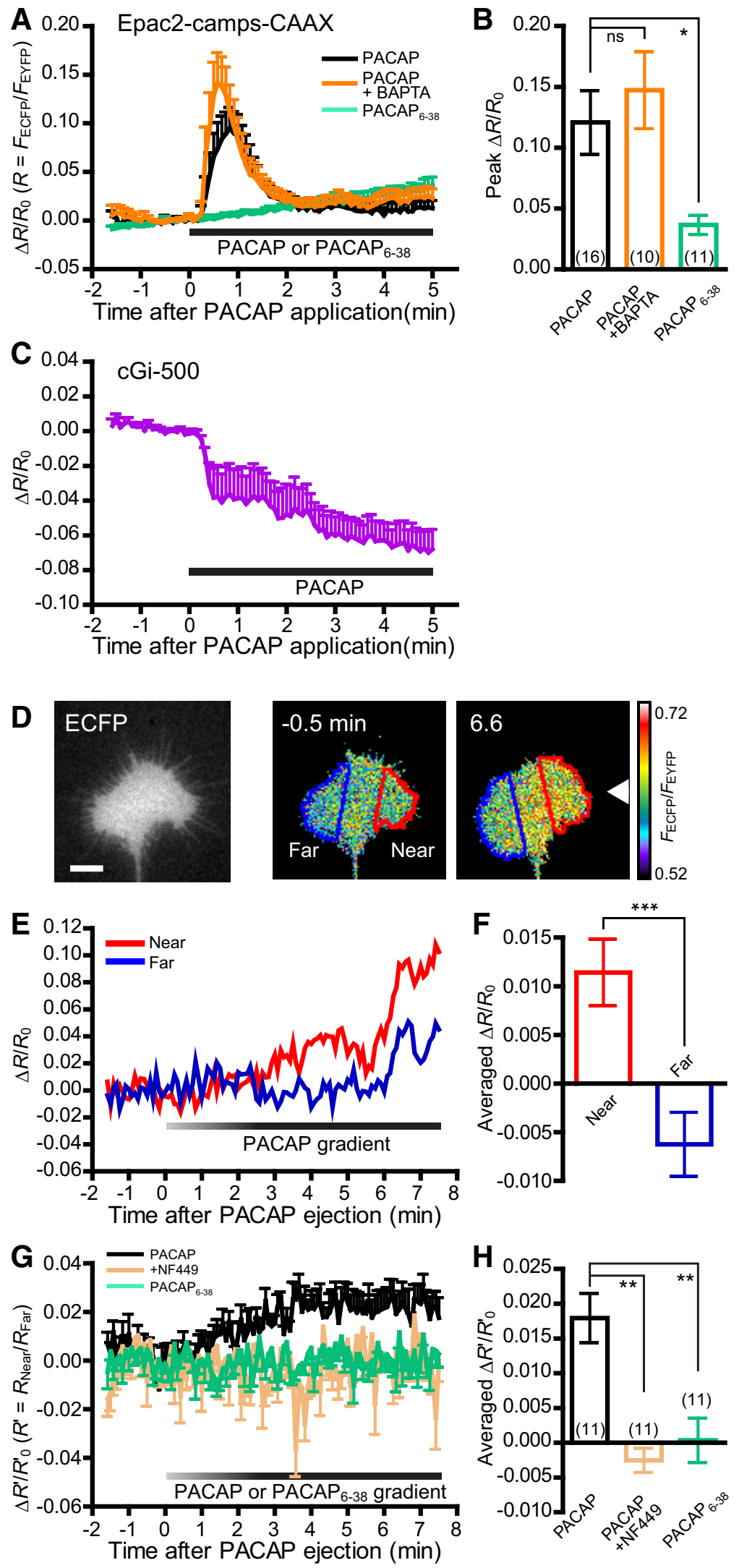

Figure 8. PACAP gradients elicit asymmetric CAMP elevation across the growth cone. $\boldsymbol{A}$, Time course changes in FRET efficiency (ECFP/EYFP emission ratio defined as $R$ ) of Epac2-camps-CAAX in growth cones exposed to bath-applied PACAP or PACAP 6 -38. The $y$-axis indicates $\Delta R / R_{0}$, where $R_{0}$ is the mean of $R$ values before the application of PACAP or PACAP ${ }_{6-38}$ and $\Delta R$ represents $R-R_{0}$. $B$, The peak $\Delta R / R_{0}$ after bath application of PACAP or PACAP ${ }_{6-38}$ in growth cones shown in $A$. Some growth cones were loaded with BAPTA-AM before PACAP application. Numbers in parentheses indicate the number of growth cones examined. * $p<0.05$; ns, cleotides (Fig. 7). Repeated FLIP of caged cAMP or cGMP on one side of growth cones induced attractive or repulsive turning, respectively (Fig. 7A,B,E). Growth cones expressing the VAMP7-LD failed to show turning responses to cAMP or cGMP signals (Fig. 7C-E). Furthermore, the shRNAmediated VAMP7 knock-down abolished cAMP-induced growth cone attraction, and this shRNA effect was rescued by the shRNA-resistant VAMP7, which retains the same amino acid sequence as wild type (Fig. $7 F, G)$. In contrast, VAMP7 shRNA did not affect $\mathrm{Ca}^{2+}$-induced attraction (Fig. 7G). We also tested for the involvement of other molecular pathways in cAMP-induced growth cone attraction. Treatment with nocodazole but not TeNT abolished cAMPinduced attraction (Fig. $7 \mathrm{H}$ ), which is consistent with our findings that cAMP facilitates MT-guided transport of VAMP7 but not VAMP2 vesicles. BAPTA-AM treatment did not affect cAMP-induced attraction (Fig. $7 \mathrm{H}$ ), indicating that cAMP can steer growth cones independently of $\mathrm{Ca}^{2+}$.

\section{cAMP activates MT-guided VAMP7 vesicle transport in cue-induced guidance}

We investigated whether cAMP facilitates MT advance and VAMP7 vesicle transport to steer growth cones in response to an extracellular guidance cue. Because most cues use both $\mathrm{Ca}^{2+}$ and cyclic nucleotides concomitantly as second messengers (Tojima et al., 2011), it is difficult to dissect cyclic nucleotide pathways in cueinduced axon guidance. Thus, we decided to study PACAP, an extracellular cue that elicits $\mathrm{Ca}^{2+}$-independent growth cone at-

\footnotetext{
not significant, unpaired or Welch's $t$ test with Holm-Bonferroni correction. C, Time course changes in FRET efficiency (ECFP/EYFP emission ratio defined as $R$ ) of $(\mathrm{Gi}-500$ in growth cones exposed to bath-applied PACAP ( $n=5$ growth cones). D, A fluorescence image of a growth cone expressing Epac2camps-CAAX. Time-lapse pseudo-color images represent $R$ before (left) and after (right) directional application of a PACAP gradient (arrowhead). ROls used to calculate $R$ on both sides of the growth cone are shown (Near and Far). Digits indicate minutes after the onset of a PACAP gradient. Scale bar, $10 \mu \mathrm{m}$. $\boldsymbol{E}$, Time course changes in $R$ in the near (red) and far (blue) ROls of the growth cone shown in $\boldsymbol{D}$. $\boldsymbol{F}$, The mean $\Delta R / R_{0}$ during the period from 1 to $7.5 \mathrm{~min}$ after the onset of PACAP gradient in the near and far ROIs ( $n=11$ growth cones). ${ }^{* * *} p<0.001$, paired $t$ test. $\mathbf{G}$, Growth cones pretreated with or without NF449 were exposed to a PACAP or PACAP ${ }_{6-38}$ gradient. Shown are time course changes in $R^{\prime}$, where $R^{\prime}$ is a ratio of $R$ in the near ROI $\left(R_{\text {Near }}\right)$ to that in the far ROI $\left(R_{\text {Far }}\right)$. Note that $R^{\prime}$ is an index of asymmetric cAMP elevations across the growth cone. $\boldsymbol{H}$, The mean $\Delta \boldsymbol{R}^{\prime} / \boldsymbol{R}^{\prime}$ in growth cones shown in $\boldsymbol{G}$ during the period from 1 to 7.5 min after the onset of PACAP or PACAP ${ }_{6-38}$ gradient. ${ }^{* *} p<0.01$, Dunnett's multiple-comparison test.
} 

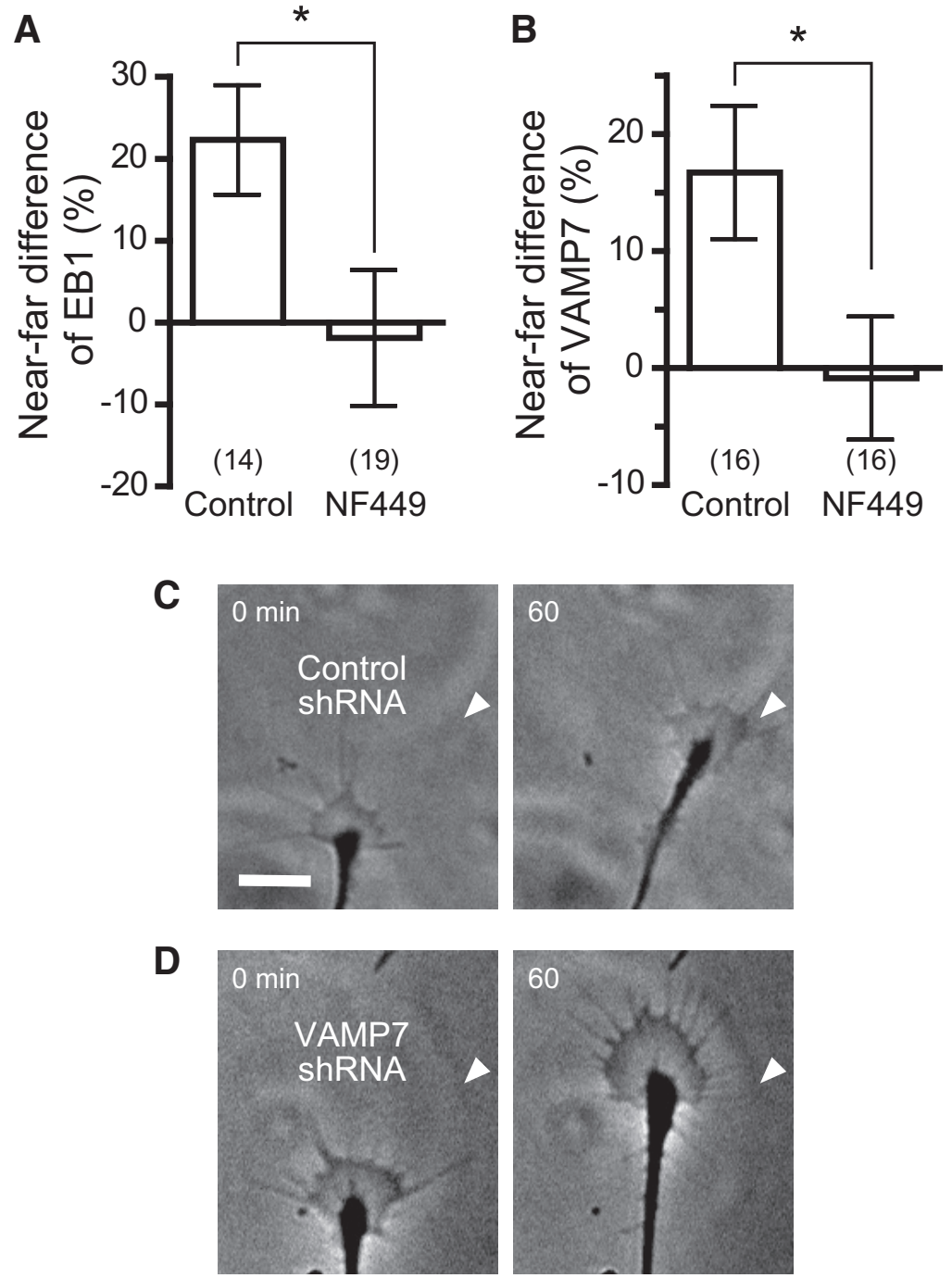

E

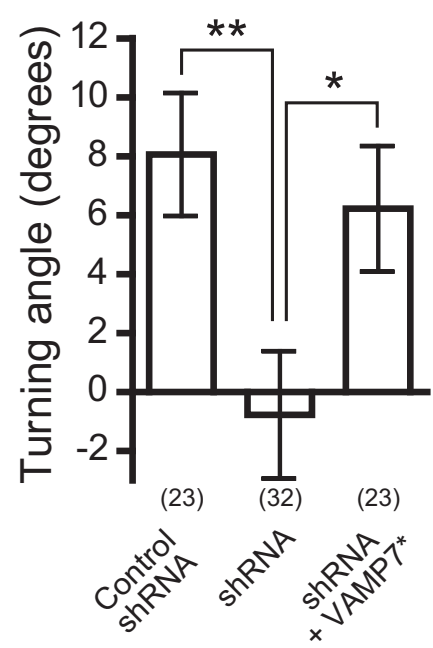

$\mathbf{F}$

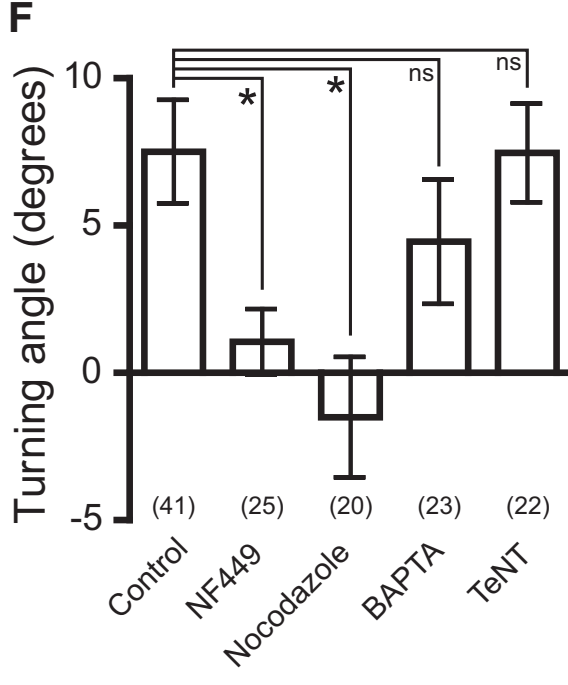

Figure 9. VAMP7-dependent membrane trafficking operates in PACAP-induced growth cone attraction. $\boldsymbol{A}$, Growth cones were exposed to PACAP gradients, and the number of EB1 comets contacting the leading edge was counted on the near and far sides. Shown is the mean of near-far differences of EB1 contact frequency calculated as (near $-\mathrm{far}) /($ near $+\mathrm{far}) \times 100$ in the absence (control) or presence of NF449. Numbers in parentheses indicate the number of growth cones examined. ${ }^{*} p<0.05$, unpaired $t$ test. $\boldsymbol{B}$, Growth cones were exposed to PACAP gradients, and the number of VAMP7 vesicles migrating centrifugally was counted on the near and far sides. Shown is the mean of near-far differences of frequency of VAMP7 vesicle centrifugal transport in the absence (control) or presence of NF449. ${ }^{*} p<0.05$, unpaired $t$ test. $\boldsymbol{C}, \boldsymbol{D}$, Time-lapse phase contrast images of growth cones treated traction by stimulating cAMP production through the G-protein-coupled receptor PAC1R (Guirland et al., 2003; Nakamachi et al., 2011). To image PACAP-elicited cAMP signaling in growth cones, we used the FRET-based cAMP sensor Epac2camps (Nikolaev et al., 2004). Because PACAP activates transmembrane adenylyl cyclase, Epac2-camps is attached to the CAAX motif of K-Ras that targets the sensor to the plasma membrane. We monitored FRET efficiency of Epac2camps-CAAX in growth cones and detected cAMP elevations in response to bath-applied PACAP but not PACAP $_{6-38}$, an inactive analog of PACAP (Fig. $8 A, B$ ). The PACAP-induced cAMP elevations were not blocked by BAPTA$\mathrm{AM}$, indicating that PACAP can activate cAMP signaling independently of $\mathrm{Ca}^{2+}$. We also monitored cGMP levels using the FRET-based cGMP sensor cGi-500 (Russwurm et al., 2007) to test whether PACAP also modulates CGMP concentrations in growth cones. PACAP reduced cGMP levels (Fig. 8C) most likely through the reciprocal inhibition pathways between cAMP and cGMP (Shelly et al., 2010, 2011). When PACAP was applied to a growth cone as an extracellular gradient, cAMP levels increased asymmetrically with more cAMP on the side of the growth cone facing the source of PACAP (Fig. 8D-F). The PACAP-induced asymmetric cAMP elevations were blocked by NF449, an inhibitor of Gs $\alpha$ signaling (Hohenegger et al., 1998). As control, PACAP $_{6-38}$ gradients caused no asymmetric increase in cAMP concentrations (Fig. 8G,H). These data demonstrate asymmetric cyclic nucleotide signaling across growth cones migrating in PACAP gradients.

We next showed that extracellular PACAP gradients increased the frequency of MT contacts with the leading edge (Fig. $9 A)$ and centrifugal transport of VAMP7 vesicles (Fig. 9B) on the side of the growth cone facing the source of PACAP. These asymmetric changes were abolished by NF449 treatment, which is consistent with our model proposing that PACAP regulates MT dynamics and VAMP7 vesicle

\section{$\leftarrow$}

with control (C) or VAMP7 shRNA (D). White arrowheads indicate the direction of PACAP gradients. Digits represent minutes after the onset of PACAP gradients. Scale bar, $10 \mu \mathrm{m} . \boldsymbol{E}, \boldsymbol{F}$, The average angles of growth cone turning induced by PACAP gradients. The growth cones were genetically or pharmacologically treated as indicated. VAMP7* is an shRNA-resistant VAMP7. $\boldsymbol{E}^{*} p<0.05 ;{ }^{* *} p<0.01$, Dunnett's multiplecomparison test; $\boldsymbol{F}_{,}^{*} p<0.05 ;$ ns, not significant, unpaired or Welch's $t$ test with Holm-Bonferroni correction. 


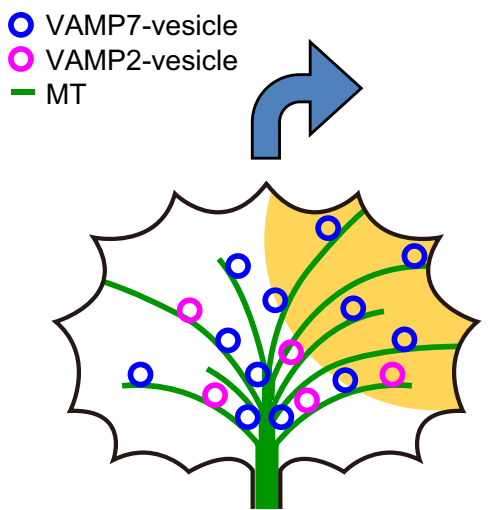

\section{CAMP signal} (Attractive)

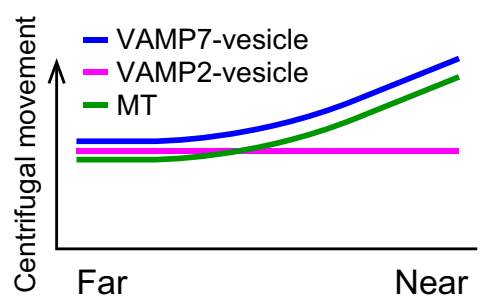

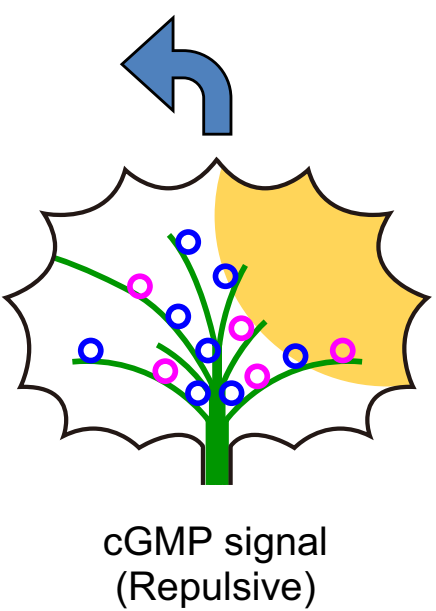

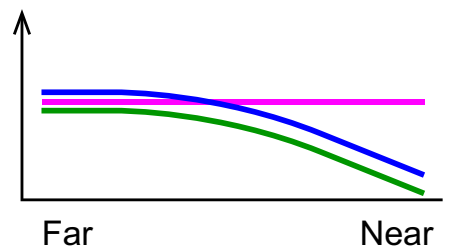

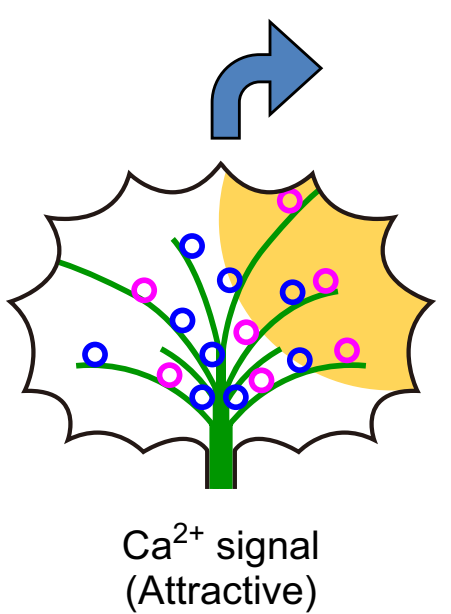

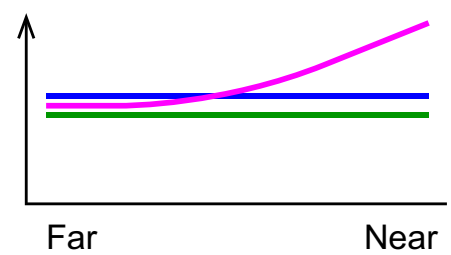

Figure 10. Schematic illustration of MT-guided membrane vesicle transport. Orange areas represent second-messenger signals mediating growth cone turning. Cyclic nucleotides counteractively regulate VAMP7 vesicle transport along polymerizing MTs: CAMP (left) and CGMP (middle) facilitates and inhibits this effector process, thereby causing attraction and repulsion, respectively. By contrast, attractive $\mathrm{Ca}^{2+}$ signals (right) promote VAMP2 vesicle transport along pre-existing MTs. Blue arrows indicate the direction of growth cone turning. The graph below each growth cone scheme shows the activity of centrifugal movement of membrane vesicles and MTs across the width of the growth cone.

transport through Gs $\alpha$-mediated production of cAMP. To confirm the involvement of VAMP7 in PACAP-induced growth cone turning, we showed that VAMP7 shRNA abolished PACAPinduced attraction and that this shRNA effect was rescued by the resistant VAMP7 (Fig. 9C-E). We further showed that PACAPinduced attraction required Gs $\alpha$ signaling and MT dynamics but not $\mathrm{Ca}^{2+}$ or its downstream VAMP2-mediated membrane trafficking (Fig. 9F), which is consistent with the previous finding that PACAP attracts growth cones via cAMP but not $\mathrm{Ca}^{2+}$ signaling (Guirland et al., 2003). Taken collectively, our results indicate that cAMP-dependent MT-guided VAMP7 vesicle transport operates in PACAP-induced growth cone attraction.

\section{Discussion}

Extracellular guidance cues activate intracellular second messengers and downstream signaling networks that control the localized protrusive activity of growth cones to navigate developing axons. While it has been assumed that MTs play an instructive role in the regulation of growth cone protrusion (Mack et al., 2000; Koester et al., 2007; Khazaei et al., 2014), no direct evidence has been provided as to whether and how MTs regulate edge membrane dynamics during growth cone guidance. Here, we report that polymerizing MTs toward the growth cone's leading edge induce localized lamellipodial protrusion by providing VAMP7-positive vesicles to the membrane. Furthermore, we have revealed that CAMP and cGMP counteractively regulate this MT-directed VAMP7 vesicle transport and resultant lamellipodial protrusion to mediate bidirectional axon guidance, which is in contrast to attractive $\mathrm{Ca}^{2+}$ signals that promote centrifugal transport of VAMP2 vesicles along pre-existing MTs. Although cyclic nucleotides and $\mathrm{Ca}^{2+}$ mediate the generation of spatial asymmetry in membrane trafficking for growth cone guidance, our studies have demonstrated distinct molecular machineries operating downstream of these second messengers (Fig. 10).

In at least two ways, VAMP7 vesicles serve as possible mechanisms for VAMP7-dependent lamellipodial protrusion during growth cone turning. First, VAMP7 vesicles act simply as suppliers of new membrane components, thereby reducing plasmalemmal tension asymmetrically across the growth cone to generate additional space for cytoskeletal polymerization (Raucher and Sheetz, 2000). Second, the vesicles facilitate asymmetric delivery and recruitment of functional molecules to growth cone plasmalemma. For example, VAMP7 vesicles carry cell adhesion molecules, such as L1, that upon exocytosis contribute to the formation of new adhesive structures necessary for edge protrusion (Alberts et al., 2003). The nonreceptor tyrosine kinase Src cooperates with the focal adhesion kinase to promote the formation of adhesive structures and actin filaments in growth cones (Robles et al., 2005; Myers and Gomez, 2011; Myers et al., 2012), and the localized activation of Src in growth cones is dependent on MT dynamics (Suter et al., 2004). In addition, membrane vesicles containing phosphatidylinositol 3,4,5trisphosphate are transported by the MT-associated kinesin motor KIF13b to the neurite tip and controls neuronal polarity (Horiguchi et al., 2006). These previous findings are consistent with our model proposing that MT advance and contact with the edge membrane induce its localized protrusion by providing functional molecules and membrane components packaged in vesicles.

Although spatial asymmetry in membrane trafficking underlies growth cone turning induced by both cyclic nucleotides and $\mathrm{Ca}^{2+}$, these second messengers activate distinct machineries for directional transport of membrane vesicles, with cyclic nucleo- 
tides acting on MT dynamics to regulate VAMP7 vesicle transport and attractive $\mathrm{Ca}^{2+}$ signals promoting the departure of VAMP2 vesicles for centrifugal transport along pre-existing MTs (Tojima et al., 2007). Because MT reorientation toward the new direction of migration accompanies any turning responses, asymmetric alterations in MT dynamics should also be observed at some point in $\mathrm{Ca}^{2+}$-mediated turning. By monitoring MT dynamics immediately after photolysis-induced activation of second-messenger pathways, we could discern distinct machineries operating at the initial phase of cyclic nucleotide-induced and $\mathrm{Ca}^{2+}$-induced growth cone turning. There are several possible pathways that can mediate the regulation of MT dynamics downstream of cyclic nucleotides. Stathmin family proteins sequester tubulin heterodimers, thereby inhibiting tubulin polymerization (Grenningloh et al., 2004). cAMP may promote MT extension by activating protein kinase A (PKA), which phosphorylates stathmin and inhibits stathmin-mediated tubulin sequestration (Grenningloh et al., 2004). PKA can also facilitate MT growth via phosphorylation of glycogen synthase kinase $3 \beta$, which regulates MT-associating proteins, such as collapsin response mediator protein 2 and adenomatous polyposis coli (Shelly et al., 2010; Kim et al., 2011).

Because VAMP7 vesicles are regarded as the major source for plasmalemmal expansion necessary for axon extension (Martinez-Arca et al., 2001), exocytic transport of this vesicle population is likely to be constitutive. This would suggest that changes in the localization of MT tips are sufficient for biasing the transport direction of VAMP7 vesicles in growth cones. Conversely, VAMP2 vesicles usually remain in the growth cone $\mathrm{C}$ domain but start centrifugal migration after attractive $\mathrm{Ca}^{2+}$ signals (Tojima et al., 2007). The departure of VAMP2 vesicles depends on specific signals and cannot be induced by MT extension as shown in this paper. We have identified a molecular pathway that initiates the departure of VAMP2 vesicles in response to attractive $\mathrm{Ca}^{2+}$ signals (Wada et al., 2016). These findings support our model proposing that alterations in MT dynamics, such as those induced by cyclic nucleotides, are sufficient to regulate the centrifugal transport of vesicles carrying VAMP7 but not VAMP2 in growth cones. A similar difference between VAMP7 and VAMP2 functions exists in mechanisms of neurotransmitter release at the synapse. While VAMP7 mediates action potential-independent spontaneous release, which is relatively insensitive to $\mathrm{Ca}^{2+}$ chelation, VAMP2 is necessary for $\mathrm{Ca}^{2+}$-evoked release (Schoch et al., 2001; Hua et al., 2011; Vyleta and Smith, 2011). Therefore, the functional difference between VAMP7 and VAMP2 may be conserved across a broad range of biological processes.

Although both cyclic nucleotides and $\mathrm{Ca}^{2+}$ participate in growth cone turning induced by many guidance cues (Tojima et al., 2011), second-messenger requirements differ among guidance cues. For example, PACAP requires cAMP but not $\mathrm{Ca}^{2+}$ for axon guidance (Guirland et al., 2003). In the present study, we examined cAMP pathways in PACAP-induced turning and showed that growth cone attraction involves VAMP7-dependent but not VAMP2-dependent membrane trafficking. By contrast, netrin-1 attracts growth cones through interplay between cAMP and $\mathrm{Ca}^{2+}$ signals (Hong et al., 2000; Nishiyama et al., 2003; Nicol et al., 2011) and mobilizes both VAMP7-dependent and VAMP2dependent trafficking (Bouchard et al., 2008; Cotrufo et al., 2011; Ros et al., 2015). These findings are consistent with our model proposing that VAMP7 and VAMP2 operate downstream of cyclic nucleotides and $\mathrm{Ca}^{2+}$, respectively, although many guidance cues are likely to activate both pathways simultaneously in growth cones.

While asymmetric increases in cyclic nucleotide concentrations in growth cones are sufficient for the induction of axon turning (Lohof et al., 1992; Guirland et al., 2003; Togashi et al., 2008), spatiotemporal dynamics of cyclic nucleotide signals have not been monitored in steering growth cones. In the present study, we used FRET-based sensors to visualize cyclic nucleotide signals in a growth cone migrating in an extracellular gradient of an attractive cue and demonstrated the generation of asymmetric cAMP signals across the growth cone with higher cAMP levels on the side facing the attractive cue. Through reciprocal inhibition pathways between cAMP and cGMP (Shelly et al., 2010, 2011; Fig. $8 C$ ), this cAMP gradient can be accompanied by a counter gradient of cGMP. We can reasonably speculate that these counter gradients of cAMP and cGMP antagonistically regulate MTdirected membrane transport, thereby increasing the growth cone sensitivity to guidance cues or improving the fidelity of axon guidance. In addition, the behavior of a growth cone exposed sequentially to multiple cues is likely to be determined through interactions between cAMP and cGMP. For example, semaphorin 3A-induced growth cone collapse depends on cGMP signal and MT dynamics (Fukata et al., 2002; Uchida et al., 2005; Togashi et al., 2008), but is attenuated if the growth cone has been pretreated with NGF, which elevates cAMP concentration (Dontchev and Letourneau, 2002). These findings are consistent with the idea that growth cone responses to guidance cues can be modulated by antagonistic activities of cAMP and cGMP, which facilitate and inhibit MT-directed membrane transport, respectively.

In summary, we have demonstrated the critical role of MTdirected membrane transport in growth cone navigation and identified cyclic nucleotides as second messengers that regulate this effector process in a counteractive manner. These findings will significantly contribute to our better understanding of molecular mechanisms of growth cone navigation and, possibly, guided cell migration in general.

\section{References}

Akhmanova A, Steinmetz MO (2008) Tracking the ends: a dynamic protein network controls the fate of microtubule tips. Nat Rev Mol Cell Biol 9:309-322. CrossRef Medline

Akiyama H, Kamiguchi H (2010) Phosphatidylinositol 3-kinase facilitates microtubule-dependent membrane transport for neuronal growth cone guidance. J Biol Chem 285:41740-41748. CrossRef Medline

Akiyama H, Kamiguchi H (2014) Analysis of calcium signals in steering neuronal growth cones in vitro. Methods Mol Biol 1162:17-27. CrossRef Medline

Akiyama H, Kamiguchi H (2015) Second messenger networks for accurate growth cone guidance. Dev Neurobiol 75:411-422. CrossRef Medline

Akiyama H, Matsu-ura T, Mikoshiba K, Kamiguchi H (2009) Control of neuronal growth cone navigation by asymmetric inositol 1,4,5trisphosphate signals. Sci Signal 2:ra34. CrossRef Medline

Alberts P, Rudge R, Hinners I, Muzerelle A, Martinez-Arca S, Irinopoulou T, Marthiens V, Tooze S, Rathjen F, Gaspar P, Galli T (2003) Cross talk between tetanus neurotoxin-insensitive vesicle-associated membrane protein-mediated transport and L1-mediated adhesion. Mol Biol Cell 14:4207-4220. CrossRef Medline

Atasoy D, Ertunc M, Moulder KL, Blackwell J, Chung C, Su J, Kavalali ET (2008) Spontaneous and evoked glutamate release activates two populations of NMDA receptors with limited overlap. J Neurosci 28:1015110166. CrossRef Medline

Bal M, Leitz J, Reese AL, Ramirez DM, Durakoglugil M, Herz J, Monteggia LM, Kavalali ET (2013) Reelin mobilizes a VAMP7-dependent synaptic vesicle pool and selectively augments spontaneous neurotransmission. Neuron 80:934-946. CrossRef Medline

Bouchard JF, Horn KE, Stroh T, Kennedy TE (2008) Depolarization recruits 
DCC to the plasma membrane of embryonic cortical neurons and enhances axon extension in response to netrin-1. J Neurochem 107: 398-417. CrossRef Medline

Buck KB, Zheng JQ (2002) Growth cone turning induced by direct local modification of microtubule dynamics. J Neurosci 22:9358-9367. Medline

Burgo A, Proux-Gillardeaux V, Sotirakis E, Bun P, Casano A, Verraes A, Liem RK, Formstecher E, Coppey-Moisan M, Galli T (2012) A molecular network for the transport of the TI-VAMP/VAMP7 vesicles from cell center to periphery. Dev Cell 23:166-180. CrossRef Medline

Cotrufo T, Pérez-Brangulí F, Muhaisen A, Ros O, Andrés R, Baeriswyl T, Fuschini G, Tarrago T, Pascual M, Ureña J, Blasi J, Giralt E, Stoeckli ET, Soriano E (2011) A signaling mechanism coupling netrin-1/deleted in colorectal cancer chemoattraction to SNARE-mediated exocytosis in axonal growth cones. J Neurosci 31:14463-14480. CrossRef Medline

Dontchev VD, Letourneau PC (2002) Nerve growth factor and semaphorin 3A signaling pathways interact in regulating sensory neuronal growth cone motility. J Neurosci 22:6659-6669. Medline

Etienne-Manneville S (2013) Microtubules in cell migration. Annu Rev Cell Dev Biol 29:471-499. CrossRef Medline

Fukata Y, Itoh TJ, Kimura T, Ménager C, Nishimura T, Shiromizu T, Watanabe H, Inagaki N, Iwamatsu A, Hotani H, Kaibuchi K (2002) CRMP-2 binds to tubulin heterodimers to promote microtubule assembly. Nat Cell Biol 4:583-591. Medline

Grenningloh G, Soehrman S, Bondallaz P, Ruchti E, Cadas H (2004) Role of the microtubule destabilizing proteins SCG10 and stathmin in neuronal growth. J Neurobiol 58:60-69. CrossRef Medline

Guirland C, Buck KB, Gibney JA, DiCicco-Bloom E, Zheng JQ (2003) Direct cAMP signaling through G-protein-coupled receptors mediates growth cone attraction induced by pituitary adenylate cyclase-activating polypeptide. J Neurosci 23:2274-2283. Medline

Hohenegger M, Waldhoer M, Beindl W, Böing B, Kreimeyer A, Nickel P, Nanoff C, Freissmuth M (1998) Gsalpha-selective G protein antagonists. Proc Natl Acad Sci U S A 95:346-351. CrossRef Medline

Hong K, Nishiyama M, Henley J, Tessier-Lavigne M, Poo M (2000) Calcium signalling in the guidance of nerve growth by netrin-1. Nature 403:93-98. CrossRef Medline

Horiguchi K, Hanada T, Fukui Y, Chishti AH (2006) Transport of PIP3 by GAKIN, a kinesin-3 family protein, regulates neuronal cell polarity. J Cell Biol 174:425-436. CrossRef Medline

Hua Z, Leal-Ortiz S, Foss SM, Waites CL, Garner CC, Voglmaier SM, Edwards RH (2011) v-SNARE composition distinguishes synaptic vesicle pools. Neuron 71:474-487. CrossRef Medline

Kamiguchi H, Yoshihara F (2001) The role of endocytic L1 trafficking in polarized adhesion and migration of nerve growth cones. J Neurosci 21: 9194-9203. Medline

Khazaei MR, Girouard MP, Alchini R, Ong Tone S, Shimada T, Bechstedt S, Cowan M, Guillet D, Wiseman PW, Brouhard G, Cloutier JF, Fournier AE (2014) Collapsin response mediator protein 4 regulates growth cone dynamics through the actin and microtubule cytoskeleton. J Biol Chem 289:30133-30143. CrossRef Medline

Kim YT, Hur EM, Snider WD, Zhou FQ (2011) Role of GSK3 signaling in neuronal morphogenesis. Front Mol Neurosci 4:48. CrossRef Medline

Koester MP, Müller O, Pollerberg GE (2007) Adenomatous polyposis coli is differentially distributed in growth cones and modulates their steering. J Neurosci 27:12590-12600. CrossRef Medline

Krause M, Gautreau A (2014) Steering cell migration: lamellipodium dynamics and the regulation of directional persistence. Nat Rev Mol Cell Biol 15:577-590. CrossRef Medline

Lohof AM, Quillan M, Dan Y, Poo MM (1992) Asymmetric modulation of cytosolic cAMP activity induces growth cone turning. J Neurosci 12: 1253-1261. Medline

Mack TG, Koester MP, Pollerberg GE (2000) The microtubule-associated protein MAP1B is involved in local stabilization of turning growth cones. Mol Cell Neurosci 15:51-65. CrossRef Medline

Martinez-Arca S, Alberts P, Zahraoui A, Louvard D, Galli T (2000) Role of tetanus neurotoxin insensitive vesicle-associated membrane protein (TI-VAMP) in vesicular transport mediating neurite outgrowth. J Cell Biol 149:889-900. CrossRef Medline

Martinez-Arca S, Coco S, Mainguy G, Schenk U, Alberts P, Bouillé P, Mezzina
M, Prochiantz A, Matteoli M, Louvard D, Galli T (2001) A common exocytotic mechanism mediates axonal and dendritic outgrowth. J Neurosci 21:3830-3838. Medline

Mimori-Kiyosue Y, Shiina N, Tsukita S (2000) The dynamic behavior of the APC-binding protein EB1 on the distal ends of microtubules. Curr Biol 10:865-868. CrossRef Medline

Myers JP, Gomez TM (2011) Focal adhesion kinase promotes integrin adhesion dynamics necessary for chemotropic turning of nerve growth cones. J Neurosci 31:13585-13595. CrossRef Medline

Myers JP, Robles E, Ducharme-Smith A, Gomez TM (2012) Focal adhesion kinase modulates Cdc42 activity downstream of positive and negative axon guidance cues. J Cell Sci 125:2918-2929. CrossRef Medline

Nakamachi T, Farkas J, Watanabe J, Ohtaki H, Dohi K, Arata S, Shioda S (2011) Role of PACAP in neural stem/progenitor cell and astrocytefrom neural development to neural repair. Curr Pharm Des 17:973-984. CrossRef Medline

Nicol X, Hong KP, Spitzer NC (2011) Spatial and temporal second messenger codes for growth cone turning. Proc Natl Acad Sci U S A 108:1377613781. CrossRef Medline

Nikolaev VO, Bünemann M, Hein L, Hannawacker A, Lohse MJ (2004) Novel single chain cAMP sensors for receptor-induced signal propagation. J Biol Chem 279:37215-37218. CrossRef Medline

Nishiyama M, Hoshino A, Tsai L, Henley JR, Goshima Y, Tessier-Lavigne M, Poo MM, Hong K (2003) Cyclic AMP/GMP-dependent modulation of $\mathrm{Ca} 2+$ channels sets the polarity of nerve growth-cone turning. Nature 423:990-995. CrossRef Medline

Ooashi N, Futatsugi A, Yoshihara F, Mikoshiba K, Kamiguchi H (2005) Cell adhesion molecules regulate $\mathrm{Ca}^{2+}$-mediated steering of growth cones via cyclic AMP and ryanodine receptor type 3. J Cell Biol 170:1159-1167. CrossRef Medline

Raucher D, Sheetz MP (2000) Cell spreading and lamellipodial extension rate is regulated by membrane tension. J Cell Biol 148:127-136. CrossRef Medline

Robles E, Woo S, Gomez TM (2005) Src-dependent tyrosine phosphorylation at the tips of growth cone filopodia promotes extension. J Neurosci 25:7669-7681. CrossRef Medline

Ros O, Cotrufo T, Martínez-MármolR, Soriano E (2015) Regulation of patterned dynamics of local exocytosis in growth cones by netrin-1. J Neurosci 35:5156-5170. CrossRef Medline

Russwurm M, Mullershausen F, Friebe A, Jäger R, Russwurm C, Koesling D (2007) Design of fluorescence resonance energy transfer (FRET)-based cGMP indicators: a systematic approach. Biochem J 407:69-77. CrossRef Medline

San Miguel-Ruiz JE, Letourneau PC (2014) The role of Arp2/3 in growth cone actin dynamics and guidance is substrate dependent. J Neurosci 34:5895-5908. CrossRef Medline

Sato Y, Kasai T, Nakagawa S, Tanabe K, Watanabe T, Kawakami K, Takahashi Y (2007) Stable integration and conditional expression of electroporated transgenes in chicken embryos. Dev Biol 305:616-624. CrossRef Medline

Schoch S, Deák F, Königstorfer A, Mozhayeva M, Sara Y, Südhof TC, Kavalali ET (2001) SNARE function analyzed in synaptobrevin/VAMP knockout mice. Science 294:1117-1122. CrossRef Medline

Shelly M, Lim BK, Cancedda L, Heilshorn SC, Gao H, Poo MM (2010) Local and long-range reciprocal regulation of cAMP and cGMP in axon/dendrite formation. Science 327:547-552. CrossRef Medline

Shelly M, Cancedda L, Lim BK, Popescu AT, Cheng PL, Gao H, Poo MM (2011) Semaphorin3A regulates neuronal polarization by suppressing axon formation and promoting dendrite growth. Neuron 71:433-446. CrossRef Medline

Suter DM, Schaefer AW, Forscher P (2004) Microtubule dynamics are necessary for SRC family kinase-dependent growth cone steering. Curr Biol 14:1194-1199. CrossRef Medline

Togashi K, von Schimmelmann MJ, Nishiyama M, Lim CS, Yoshida N, Yun B, Molday RS, Goshima Y, Hong K (2008) Cyclic GMP-gated CNG channels function in Sema3A-induced growth cone repulsion. Neuron 58: 694-707. CrossRef Medline

Tojima T, Akiyama H, Itofusa R, Li Y, Katayama H, Miyawaki A, Kamiguchi $\mathrm{H}$ (2007) Attractive axon guidance involves asymmetric membrane transport and exocytosis in the growth cone. Nat Neurosci 10:58-66. CrossRef Medline 
Tojima T, Itofusa R, Kamiguchi H (2009) The nitric oxide-cGMP pathway controls the directional polarity of growth cone guidance via modulating cytosolic $\mathrm{Ca}^{2+}$ signals. J Neurosci 29:7886-7897. CrossRef Medline

Tojima T, Itofusa R, Kamiguchi H (2010) Asymmetric clathrin-mediated endocytosis drives repulsive growth cone guidance. Neuron 66:370-377. CrossRef Medline

Tojima T, Hines JH, Henley JR, Kamiguchi H (2011) Second messengers and membrane trafficking direct and organize growth cone steering. Nat Rev Neurosci 12:191-203. CrossRef Medline

Tojima T, Itofusa R, Kamiguchi H (2014) Steering neuronal growth cones by shifting the imbalance between exocytosis and endocytosis. J Neurosci 34:7165-7178. CrossRef Medline

Uchida Y, Ohshima T, Sasaki Y, Suzuki H, Yanai S, Yamashita N, Nakamura F, Takei K, Ihara Y, Mikoshiba K, Kolattukudy P, Honnorat J, Goshima Y (2005) Semaphorin3A signalling is mediated via sequential Cdk5 and GSK3beta phosphorylation of CRMP2: implication of common phos- phorylating mechanism underlying axon guidance and Alzheimer's disease. Genes Cells 10:165-179. CrossRef Medline

Vyleta NP, Smith SM (2011) Spontaneous glutamate release is independent of calcium influx and tonically activated by the calcium-sensing receptor. J Neurosci 31:4593-4606. CrossRef Medline

Wada F, Nakata A, Tatsu Y, Ooashi N, Fukuda T, Nabetani T, Kamiguch H (2016) Myosin Va-Endoplasmic Reticulum Calcium Channel Complex Regulates Membrane Export during Axon Guidance. Cell Rep, in press.

Zhou FQ, Waterman-Storer CM, Cohan CS (2002) Focal loss of actin bundles causes microtubule redistribution and growth cone turning. J Cell Biol 157:839-849. CrossRef Medline

Zylbersztejn K, Petkovic M, Burgo A, Deck M, Garel S, Marcos S, BlochGallego E, Nothias F, Serini G, Bagnard D, Binz T, Galli T (2012) The vesicular SNARE synaptobrevin is required for semaphorin 3A axonal repulsion. J Cell Biol 196:37-46. CrossRef Medline 